\title{
Recent Developments in Empirical Likelihood ANd Related Methods
}

\author{
Paulo M.D.C. Parente \\ Department of Economics \\ University of Exeter \\ and \\ CReMic \\ Faculty of Economics \\ University of Cambridge \\ e-mail: p.m.parente@exeter.ac.uk
}

\author{
Richard J. Smith \\ cemmap \\ U.C.L and I.F.S. \\ and \\ Faculty of Economics \\ University of Cambridge \\ e-mail: rjs27@econ.cam.ac.uk
}

This Draft: August 2013

\begin{abstract}
This article reviews a number of recent contributions to estimation and inference for models defined by moment condition restrictions. The particular emphasis is on the generalised empirical likelihood class of estimators as an alternative to generalised method of moments. Estimation methods for parameters defined through moment restrictions and their properties are described with tests of overidentifying moment restrictions and parametric hypotheses. Computational issues are discussed together with some proposals for their amelioration. Higher order and other properties are also addressed in some detail. Models specified by conditional moment restriction models are considered and a discussion of the adaptation of these methods to weakly dependent data is given.
\end{abstract}

Key-words: Moment Conditions, GMM, Minimum Discrepancy, Generalised Minimum Contrast, Generalised Empirical Likelihood.

Corresponding author: Richard J. Smith, Faculty of Economics, University of Cambridge, Austin Robinson Building, Sidgwick Avenue, Cambridge CB3 9DD, UK. E-mail address: rjs27@econ.cam.ac.uk (R.J. Smith) 


\section{Article Table of Contents}

\section{INTRODUCTION}

MOMENT CONDITIONS

ESTIMATION METHODS

- Efficient GMM

- Empirical Likelihood

- Minimum Discrepancy

- GEL

TESTS

- Over-Identifying Moment Conditions

- Parametric Restrictions

- Nuisance Parameters

COMPUTATION

HIGHER ORDER PROPERTIES

- Asymptotic Bias

- Bartlett Correction

- Large Deviations

- Robustness

CONDITIONAL MOMENTS

- Approximating Functions 
- Conditional (G)EL

- Unknown Functions

WEAKLY DEPENDENT DATA

- Efficient (G)EL

- Higher Order Properties

CONCLUSIONS

LITERATURE CITED 


\section{INTRODUCTION}

Many if not most estimators commonly employed in empirical economic research may be motivated and formulated as the solution to a suitably defined set of moment restrictions. Indeed, the least squares estimator in the standard linear regression model is expressed in terms of the requirement that the sample covariance or correlation between regression residuals and the regressors is zero. Under correct specification, the parameters of the linear regression model are defined by the population counterpart of this condition. Hence, these moment estimators may be regarded as analogue estimators being based on the sample counterparts of population moment conditions; see Manski (1988). The maximum likelihood (ML) estimator is a further example that solves the likelihood equations, i.e., sets the first order conditions or score vector to zero, with its population version being the zero expectation of the score; see, e.g., chapter 12 in Goldberger (1991). A particular feature of these examples is that the number of parameters is identical to the number of moment restrictions, i.e., the model is just-identified.

The models economists are often concerned with typically include explanatory variables that are themselves endogenous, e.g., in the regression context when the dependent and one or more of the regressor variables or covariates are jointly determined. This may arise because of omission of relevant variables, measurement error or the economic model under investigation stipulating simultaneous determination. In such circumstances, in the regression context, the standard approach is to seek instruments or instrumental variables that are correlated with regressor variables but are uncorrelated with the regression error. The moment condition for estimation is then described by the covariance or correlation between regression error and instruments being zero. The number of instruments and, thus, moment conditions, may exceed the number of parameters to be estimated yielding an over-identified model. Consequently the sample analogue of the moment restrictions cannot no longer be used directly for parameter estimation.

The standard approach to deal with an over-identified model minimises a distance measure expressed in terms of the sample covariance between the regression errors and 
instruments. Of course, the resultant estimator will depend on the definition of distance adopted. For computational purposes a weighted Euclidean distance is often convenient. A particular choice for the weight matrix results in the instrumental variable estimator introduced in Wright (1925), Wright (1928) and Reiersol (1941, 1945); see also Sargan (1958, 1959). This approach was extended to endogenous non-linear regression models in Amemyia (1974). Hansen (1982) considers a general set-up with non-linear moment restrictions and introduces the generalised method of moments (GMM) estimation procedure. GMM is asymptotically efficient if the weight matrix is chosen as the inverse of the variance matrix of the sample moments. Given an initial consistent parameter estimator this variance matrix may be straightforwardly estimated with the resultant two-step (2S) GMM estimator being asymptotically efficient.

There is now extensive simulation evidence that the statistical properties of 2SGMM for the sample sizes typically available to the empirical investigator can be quite unsatisfactory which has stimulated intensive interest in other methods of estimation for moment condition models. Empirical likelihood (EL) was originally proposed in Owen (1988) for the estimation of a population mean and was introduced independently by Qin and Lawless (1994) and Imbens (1997) for models specified by the type of moment conditions considered in Hansen (1982). EL differs from GMM in that it is a non-parametric likelihood method of estimation based on a multinomial density formulation that incorporates the moment conditions as restrictions. Consequently EL displays obvious similarities to classical maximum likelihood (ML) for fully parametric models. ${ }^{1}$

As is well-known classical ML possesses a number of optimality and other useful

\footnotetext{
${ }^{1}$ Evidence presented in the Special Section, July 1996, of J. Bus. Econ. Stat. indicates that 2SGMM may be severely biased and initiated interest in alternative estimation methods. Most papers concerned with moment condition models such as those considered in this review investigate via Monte Carlo studies finite sample mean and median estimator biases. Ramalho (2005) considers covariance structure models and although estimators cannot be ranked in terms of mean bias median bias is lower for EL and related estimators than for 2SGMM. Guggenberger (2008) corroborates these results but observes that the standard deviation of EL and other estimators appear very large suggesting that these estimators may not possess finite sample moments. Kitamura (2007) also studies mean and median bias of several estimators in a dynamic panel data model, a similar design to that analysed in Imbens (2002); the conclusions are rather similar to those of Ramalho (2005) and Guggenberger (2008). See also the studies in Imbens and Spady (2005), Mittlehammer et al. (2005) and Newey et al. (2005).
} 
properties. First, ML is asymptotically efficient, i.e., the asymptotic variance of the ML estimator coincides with the Cramer-Rao lower bound for regular estimators. Secondly, bias-corrected ML is higher order efficient; see Ghosh (1994). Thirdly, the likelihood ratio (LR) test based on ML is optimal with respect to a large deviation optimality criterion; see Hoeffding (1965). Finally, Bartlett (1937) showed that the LR statistic for a test of a simple hypothesis may be scale-corrected by what is now commonly referred to as a Bartlett correction thereby ensuring a more rapid rate of convergence in distribution to the chi-square distribution than the original LR statistic. This result has subsequently been extended to more general forms of parametric hypotheses; see, e.g., Cribari-Neto and Cordeiro (1996) and the references therein.

Given its similarity to classical ML perhaps it is unsurprising that EL also possesses some desirable and similar properties. Qin and Lawless (1994) and Imbens (1997) show that EL shares the asymptotically efficiency property of 2SGMM. Newey and Smith (2004), henceforth NS, prove that bias corrected EL is higher order efficient. Kitamura (2001) and Kitamura et al. (2012) demonstrate that the EL criterion function test statistic for over-identifying moment conditions is optimal with respect to a large deviation optimality criterion. Chen and Cui (2006, 2007) and Matsushita and Otsu (2013) show that the EL criterion function test statistic for parametric restrictions and over-identifying moment conditions is Bartlett correctable.

A number of alternatives to EL have also been proposed that share its first order efficiency properties including exponential tilting (ET), see Kitamura and Stutzer (1997) and Imbens et al. (1998), and the continuous updating estimator (CUE), see Hansen, Heaton, and Yaron (1996). Corcoran (1998) introduced a class of estimators that minimises a discrepancy measure between the empirical distribution function and the empirical distribution function constrained to satisfy the moment restrictions in the sample, the latter thereby constituting a saddle point problem. Several estimators belong to this class, including EL, ET, CUE and those based on members of the Cressie-Read (1984) (CR) class of divergence measures proposed by Baggerly (1998). Kitamura (2007) considers a general class of $f$-divergence measures as defined in Csiszar (1963) and Ali and Silvey 
(1966) and defines the generalised minimum contrast (GMC) class of estimators.

A different class of estimators is proposed in Smith $(1997,2011)$ motivated as a non-parametric adaptation to the moment condition setting of the approach taken in Chesher and Smith (1997) which develops LR tests for parametric moment conditions in the likelihood framework. Although it differs from GMC the generalised empirical likelihood (GEL) class of estimators also requires the solution of a saddle point problem and includes EL, ET, CUE and the CR class as special cases; see Smith (1997) and NS. NS proves that GEL contains a subclass of estimators that shares the same asymptotic bias as EL and, if this GEL subclass is restricted further, bias corrected GEL, like bias corrected EL, is higher order efficient.

The review is organised as follows. Section 2 describes the moment condition framework. Estimation methods for parameters defined through moment restrictions are given in section 3. Tests of over-identifying moment restrictions and parametric hypotheses are presented in section 4 . In practice although the large sample properties of $(\mathrm{G}) \mathrm{EL}$ are attractive computation may prove to be difficult in comparison to GMM since (G)EL solves a saddle point problem. Section 5 discusses this issue and some proposals for their amelioration. Higher order and other properties of GEL are addressed in some detail in section 6 . Section 7 deals with conditional moment restriction models whereas section 8 considers how (G)EL, originally designed for the cross-section context, may be suitably adapted for weakly dependent data. Section 9 concludes with a brief outline of some open research areas together with a short discussion of other topics that could not be addressed in this review because of space constraints. Useful additional references on (G)EL are Anatolyev and Gospodinov (2011), Imbens (2002) and Kitamura (2007).

\section{MOMENT CONDITIONS}

This section outlines the general framework used in this review. A number of empirically relevant examples are provided as illustrations.

Let $z$ denote a vector of $d_{z}$ observable random variables. To describe the moment 
condition framework requires let $g(z, \beta)=\left(g^{1}(z, \beta), \ldots, g^{m}(z, \beta)\right)^{\prime}$ denote the moment indicator vector, a $m$-vector of known functions of the data vector $z$ and the $p$-vector of parameters $\beta$ which is of particular inferential interest to the investigator. The dimension $m$ of the moment indicator vector is at least as great as $p$ that of the parameter vector $\beta$, i.e., the model is either just-identified $m=p$ or over-identified $m>p$. It is assumed that the moment conditions

$$
\mathbb{E}[g(z, \beta)]=0
$$

are uniquely satisfied when $\beta$ takes the unknown true value $\beta_{0}$. Here $\mathbb{E}[\cdot]$ denotes expectation taken with respect to the distribution of $z$.

A number of well-known estimation problems fall within this setting.

EXAMPLE 2.1 (Maximum Likelihood.) Suppose that $z$ is distributed with probability density function $f(z, \beta)$ twice differentiable in $\beta$. It is assumed that although the function form of $f(z, \beta)$ is known the parameter vector $\beta_{0}$ is not. The score vector $s(z, \beta)$ is the first order derivative of the logarithm of the density $f(z, \beta)$, i.e., $s(z, \beta)=\partial \log f(z, \beta) / \partial \beta$, where $\log (\cdot)$ is the natural $\operatorname{logarithm}$. It may be shown straightforwardly that

$$
\mathbb{E}\left[s\left(z, \beta_{0}\right)\right]=0
$$

where in this example $\mathbb{E}[\cdot]$ denotes expectation taken with respect to $f\left(z, \beta_{0}\right)$; see, e.g., Goldberger (1991, p.128).

EXAMPLE 2.2 (Quantile Regression.) Let $z=\left(y, x^{\prime}\right)^{\prime}$ where $x$ is a random vector of dimension $d_{x}=d_{z}-1$. The $\theta$-quantile regression model is defined by the probability statement $\mathcal{P}\left\{y \leq x^{\prime} \beta_{0}\right\}=\theta$. The moment condition (2.1) defining the standard quantile regression estimator is given by

$$
\mathbb{E}\left[x\left(\theta-I\left(y \leq x^{\prime} \beta_{0}\right)\right)\right]=0,
$$

where the indicator function $I(A)=1$ if $A$ is true and 0 otherwise. Here $p=d_{x}$. 
Examples 2.1 and 2.2 correspond to just-identified moment condition models since $m=p$ in both cases. The next example allows for under- $m<p$, just- $m=p$ and over-identification $m>p$ as possibilities.

EXAMPLE 2.3 (Instrumental Variables.) In this example the observation vector $z$ is redefined as $z=\left(y, x^{\prime}, w^{\prime}\right)^{\prime}$ where $w$ is a random $d_{w^{-v}}$ vector of instruments or instrumental variables that satisfies the moment condition

$$
\mathbb{E}\left[w\left(y-x^{\prime} \beta_{0}\right)\right]=0,
$$

i.e., the standard instrument validity condition $\mathbb{E}[w u]=0$ in which the regression error $u=y-x^{\prime} \beta_{0}$ is uncorrelated with the vector of instruments $w$; cf. (2.1). Here, as in Example 2.2, $p=d_{x}$ but now $m=d_{w}$.

\section{ESTIMATION METHODS}

In this section, $z_{i},(i=1, \ldots, n)$, denotes a random sample of data observations drawn from the distribution of $z$. Then, for given $\beta$, the sample analog of the population expectation $\mathbb{E}[g(z, \beta)]$ is given by the sample mean $\hat{g}(\beta)=\sum_{i=1}^{n} g_{i}(\beta) / n$, where $g_{i}(\beta)=$ $g\left(z_{i}, \beta\right),(i=1, \ldots, n)$. Additionally, let $\Omega(\beta)=\mathbb{E}\left[g(z, \beta) g(z, \beta)^{\prime}\right]$ and $\Omega=\Omega\left(\beta_{0}\right)$ the positive definite variance matrix of $g\left(z, \beta_{0}\right)$; the sample counterpart of $\Omega(\beta)$ is denoted by $\hat{\Omega}(\beta)=\sum_{i=1}^{n} g_{i}(\beta) g_{i}(\beta)^{\prime} / n$. It is assumed in the following that the moment indicator vector $g(z, \beta)$ is first order differentiable with respect to $\beta$ with the consequent definitions of the full rank population Jacobian matrix $G=G\left(\beta_{0}\right)$, where $G(\beta)=\mathbb{E}\left[\partial g(z, \beta) / \beta^{\prime}\right]$, and the sample Jacobian $\hat{G}(\beta)=\sum_{i=1}^{n} G_{i}(\beta) / n$ where $G_{i}(\beta)=\partial g_{i}(\beta) / \partial \beta^{\prime},(i=1, \ldots, n)$.

We use the common notation $\hat{\beta}$ for all efficient estimators of $\beta_{0}$ described below since, under suitable conditions, they share the same first order large sample properties, i.e., consistency, $\hat{\beta} \stackrel{p}{\rightarrow} \beta_{0}$, root- $n$ asymptotic normality and first order asymptotic efficiency $n^{1 / 2}\left(\hat{\beta}-\beta_{0}\right) \stackrel{d}{\rightarrow} N(0, \Sigma)$, where $\Sigma=\left(G^{\prime} \Omega^{-1} G\right)^{-1}$ is the semiparametric efficiency lower bound [Chamberlain (1987)]. 


\subsection{Efficient GMM}

That the moment condition $\mathbb{E}[g(z, \beta)]=0(2.1)$ is satisfied uniquely at $\beta=\beta_{0}$ and that the sample mean $\hat{g}(\beta)$ should closely approximate the population mean $\mathbb{E}[g(z, \beta)]$ uniformly in $\beta$ for all $n$ sufficiently large suggest that an appropriate estimator of $\beta_{0}$ should minimise some measure of distance between $\hat{g}(\beta)$ and 0 . These arguments motivate the GMM estimator originally proposed in Hansen (1982)

$$
\tilde{\beta}=\arg \min _{\beta \in \mathcal{B}} \hat{g}(\beta)^{\prime} \hat{W}^{-1} \hat{g}(\beta)
$$

where $\mathcal{B}$ denotes the parameter space and $\hat{W}$ is a positive semi-definite matrix such that $\hat{W}$ converges in probability to the positive definite matrix $W$.

Hansen (1982) showed that, under certain regularity conditions, the GMM estimator

$\tilde{\beta}$ is consistent for $\beta_{0}$ and asymptotically normally distributed with asymptotic variance matrix given by avar $[\tilde{\beta}]=\left(G^{\prime} W^{-1} G\right)^{-1} G^{\prime} W^{-1} \Omega W^{-1} G\left(G^{\prime} W^{-1} G\right)^{-1}$, i.e., $n^{1 / 2}\left(\tilde{\beta}-\beta_{0}\right)$ converges in distribution to a $N\left(0,\left(G^{\prime} W^{-1} G\right)^{-1} G^{\prime} W^{-1} \Omega W^{-1} G\left(G^{\prime} W^{-1} G\right)^{-1}\right)$ distributed random vector. Additionally, among the class of GMM estimators defined by (3.1), the efficient GMM estimator sets $W=\Omega$. Given an initial consistent GMM estimate $\tilde{\beta}$ for $\beta_{0}$, e.g., obtained by setting $\hat{W}=I_{m}$, then, an efficient 2SGMM estimator results from replacing $\hat{W}$ by $\hat{\Omega}(\tilde{\beta})$ in $(3.1)$, i.e.,

$$
\hat{\beta}=\arg \min _{\beta \in \mathcal{B}} \hat{g}(\beta)^{\prime} \hat{\Omega}(\tilde{\beta})^{-1} \hat{g}(\beta)
$$

with asymptotic variance matrix $\Sigma=\left(G^{\prime} \Omega^{-1} G\right)^{-1}$. The matrices $G$ and $\Omega$ may be consistently estimated by $\hat{G}(\hat{\beta})$ and $\hat{\Omega}(\hat{\beta})$.

\subsection{Empirical Likelihood}

Owen (1988) originally proposed EL to define confidence regions for the population mean and differentiable functionals of the mean; see also Owen (1990) and the monograph Owen (2001). A generalisation of EL to models specified by moment conditions of the form (2.1) was provided in Qin and Lawless (1994) and Imbens (1997). 
Essentially EL is a non-parametric generalisation of parametric ML to the moment condition setting; indeed if $z$ is a vector of discrete distributed random variables EL is ML. EL treats the data as if it were discrete with probabilities $\pi_{i},(i=1, \ldots, n)$, assigned to each sample point and like ML EL estimates these probabilities so as to maximise the probability of observing the sample but subject to the imposition of the additional condition that the moment conditions are satisfied. To describe EL consider the multinomial log-likelihood

$$
\sum_{i=1}^{n} \log \pi_{i}
$$

The EL estimator of $\beta_{0}$ maximises the criterion (3.3) subject to the unit simplex definitional constraint on the probabilities $\pi_{i},(i=1, \ldots, n)$, i.e., nonnegativity $\pi_{i} \geq 0$, $(i=1, \ldots, n)$, and unit summability $\sum_{i=1}^{n} \pi_{i}=1$, together with the moment restriction $\sum_{i=1}^{n} \pi_{i} g_{i}(\beta)=0$, cf. (2.1). After profiling out the probabilities $\pi_{i},(i=1, \ldots, n)$, and the Lagrange multiplier associated with the unit summability constraint, the EL criterion is

$$
\mathcal{E} \mathcal{L}_{n}(\beta, \lambda)=\sum_{i=1}^{n} \log \left(1+\lambda^{\prime} g_{i}(\beta)\right) / n
$$

where $\lambda$ is the Lagrange multiplier associated with the sample moment constraint $\sum_{i=1}^{n} \pi_{i} g_{i}(\beta)=$ 0 . The EL estimator satisfies

$$
\hat{\beta}=\arg \min _{\beta \in \mathcal{B}} \sup _{\lambda \in \hat{\Lambda}_{n}(\beta)} \mathcal{E} \mathcal{L}_{n}(\beta, \lambda)
$$

where $\hat{\Lambda}_{n}(\beta)=\left\{\lambda: \lambda^{\prime} g_{i}(\beta)>-1, i=1, \ldots, n\right\}$ ensuring the log function is well defined. The non-negativity restriction is thus automatically satisfied since the estimated, typically referred to as implied or empirical, probabilities are given by

$$
\hat{\pi}_{i}=\frac{1}{n\left(1+\hat{\lambda}^{\prime} g_{i}(\hat{\beta})\right)},(i=1, \ldots, n)
$$

the Lagrange multiplier estimator is $\hat{\lambda}=\arg \max _{\lambda \in \hat{\Lambda}_{n}(\hat{\beta})} \sum_{i=1}^{n} \log \left(1+\lambda^{\prime} g_{i}(\hat{\beta})\right)$. 


\subsection{Minimum Discrepancy}

Corcoran (1998) introduced a class of estimators based on the minimization of a discrepancy measure defined by

$$
\mathcal{I}\left(\pi^{n}, \iota^{n}\right),
$$

where $\pi^{n}=\left(\pi_{1}, \ldots, \pi_{n}\right)^{\prime}$ and $\iota^{n}$ is an $n$-vector with all elements equal to the unrestricted empirical probabilities $1 / n$. Minimum discrepancy (MD) estimators minimise (3.6) with respect to $\pi^{n}$ and subject to $\pi_{i} \geq 0,(i=1, \ldots, n), \sum_{i=1}^{n} \pi_{i}=1$ and $\sum_{i=1}^{n} \pi_{i} g_{i}(\beta)=0$, cf., section 3.2. Several estimators belong to this class, in particular, EL, continuous updating (CUE) [Hansen et al. (1996)], exponential tilting (ET) [Kitamura and Stutzer (1997) and Imbens et al. (1998)] and the Cressie-Read (CR) class of estimators [Cressie and Read (1984)].

Kitamura (2007) recently suggested the use of $f$-divergence, see Csiszar (1963) and Ali and Silvey (1966), to define the Generalised Minimum Contrast (GMC) class of estimators. ${ }^{2}$ Here the discrepancy measure in (3.6) is redefined as

$$
\begin{aligned}
\mathcal{I}\left(\pi^{n}, \iota^{n}\right) & =\sum_{i=1}^{n} \iota_{i} \phi\left(\pi_{i} / \iota_{i}\right) \\
& =\frac{1}{n} \sum_{i=1}^{n} \phi\left(n \pi_{i}\right),
\end{aligned}
$$

where $\iota_{i}=1 / n,(i=1, \ldots, n)$, and $\phi(\cdot)$ is a convex function defined on the half-line $[0, \infty)$ and continuous at zero such that $\phi(1)=0$. For EL $\phi(v)=-\log v$, ET $\phi(v)=v \log v$, $\operatorname{CUE} \phi(v)=(v-1)^{2} / 2$ and for $\mathrm{CR} \phi(v)=\left[v^{\tau+1}-v-\tau(v-1)\right] /[\tau(1+\tau)]$. The nonnegativity condition $\pi_{i} \geq 0,(i=1, \ldots, n)$, essential for their interpretation as empirical probabilities, is often ignored in practice for CUE and CR although it must be imposed for EL, see section 3.2, and is automatically satisfied for ET. Kitamura (2007) shows that the dual of (3.6) is given by

$$
\hat{\beta}=\arg \min _{\beta \in \mathcal{B}} \sup _{\gamma, \lambda} \sum_{i=1}^{n}\left(\gamma-\frac{1}{n} \phi^{*}\left(\gamma+\lambda^{\prime} g_{i}(\beta)\right)\right),
$$

\footnotetext{
${ }^{2}$ The $f$-divergence between two discrete probability distributions $p=\left\{p_{1}, p_{2}, \ldots\right\}$ and $q=\left\{q_{1}, q_{2}, \ldots\right\}$ is defined by $D_{\phi}(p, q)=\sum_{i=1}^{\infty} p_{i} \phi\left(q_{i} / p_{i}\right)$ where the function $\phi(\cdot)$ is defined below.
} 
where $\phi^{*}(v)=\sup _{x}[x v-\phi(x)]$ is the Legendre transform of $\phi(\cdot)$. It follows that for $\mathrm{EL} \phi^{*}(v)=-1-\log (-v), \operatorname{ET} \phi^{*}(v)=\exp (v-1), \mathrm{CUE} \phi^{*}(v)=v^{2} / 2+v$ and $\mathrm{CR}$ $\phi^{*}(v)=(\tau v+1)^{1 / \tau+1} /(\tau+1)-1 /(\tau+1)$.

\subsection{GEL}

GEL, introduced in Smith (1997), also see Smith (2011), differs in general from MD and GMC. ${ }^{3}$ Although it is not explicitly defined in terms of a program based on empirical probabilities, GEL like them also includes EL, ET, CUE and the CR class of estimators as special cases.

The GEL class of estimators is defined as follows. Let

$$
\hat{P}_{n}^{\rho}(\beta, \lambda)=\sum_{i=1}^{n}\left[\rho\left(\lambda^{\prime} g_{i}(\beta)\right)-\rho_{0}\right] / n,
$$

where the function $\rho(\cdot)$ is concave on its domain $\mathcal{V}$, an open interval containing zero, with derivatives $\rho_{j}(v)=\partial^{j} \rho(v) / \partial \nu^{j}, \rho_{j}(0)=\rho_{j},(j=0,1, \ldots)$, normalised without loss of generality as $\rho_{1}=\rho_{2}=-1$. The GEL estimator of $\beta_{0}$ is given by

$$
\hat{\beta}=\arg \min _{\beta \in \mathcal{B}} \sup _{\lambda \in \hat{\Lambda}_{n}(\beta)} \hat{P}_{n}^{\rho}(\beta, \lambda)
$$

where $\hat{\Lambda}_{n}(\beta)=\left\{\lambda: \lambda^{\prime} g_{i}(\beta) \in \mathcal{V}, i=1, \ldots, n\right\}$ with the Lagrange multiplier-like estimator $\hat{\lambda}=\arg \max _{\lambda \in \hat{\Lambda}_{n}(\hat{\beta})} \hat{P}_{n}^{\rho}(\hat{\beta}, \lambda)$ the first order condition for which imposes the sample moment constraint $\sum_{i=1}^{n} \hat{\pi}_{i} g_{i}(\hat{\beta})=0$; cf. section 3.2. The implied GEL empirical probabilities $\hat{\pi}_{i},(i=1, \ldots, n)$, are

$$
\hat{\pi}_{i}=\frac{\rho_{1}\left(\hat{\lambda}^{\prime} \hat{g}_{i}\right)}{\sum_{j=1}^{n} \rho_{1}\left(\hat{\lambda}^{\prime} \hat{g}_{j}\right)},(i=1, \ldots, n),
$$

summing to one by construction, but are typically not all non-negative in finite samples, where $\hat{g}_{i}=g_{i}(\hat{\beta}),(i=1, \ldots, n) .{ }^{4}$ For any function $a(z, \beta)$ a semiparametrically

\footnotetext{
${ }^{3}$ Smith $(1997,2011)$ motivate GEL as a nonparametric generalisation to the moment condition context of the approach taken in Chesher and Smith (1997) in a fully parametric likelihood setting. Chesher and Smith (1997) proposes likelihood ratio test statistics for implied moment conditions where the likelihood augments the null hypothesis parametric density multiplicatively by a function of a weighted version of the moment indicators underpinning the implied moment conditions.

${ }^{4}$ The shrinkage estimators $\tilde{\pi}_{i}=\left(\hat{\pi}_{i}+n^{-1} \varepsilon_{n}\right) /\left(1+\varepsilon_{n}\right), \quad(i=1, \ldots, n)$, where $\varepsilon_{n}=$
} 
efficient estimator of the moment $\mathbb{E}\left[a\left(z, \beta_{0}\right)\right]$ is formed from the empirical probabilities as $\sum_{i=1}^{n} \hat{\pi}_{i} a\left(z_{i}, \hat{\beta}\right) ;$ see Brown and Newey (1998).

As noted above GEL does not coincide with MD or GMC. Since GEL is the dual of the $f$-divergence program for the CR class that includes EL, ET and CUE [Smith (1997) and NS], GEL therefore yields the same estimators for this class, see Kitamura (2007). NS, Kitamura (2007) and Smith (2007c) note that this result holds if the inverse of $\phi(\cdot)$ defining the GMC class (3.7) is homogeneous. GEL includes EL: $\rho(v)=\log (1+v)$ and $\mathcal{V}=(-1,+\infty)$, ET: $\rho(v)=-\exp (v)$, CUE: $\rho(v)=-(v+1)^{2} / 2$ and $\mathrm{CR}: \rho(v)=$ $-(1+\tau v)^{(1+\tau) / \tau} /(1+\tau)$.

NS obtained the joint limit distribution of $\hat{\beta}$ and $\hat{\lambda}$ as

$$
n^{1 / 2}\left(\begin{array}{c}
\hat{\beta}-\beta_{0} \\
\hat{\lambda}
\end{array}\right) \stackrel{d}{\rightarrow} N(0, \operatorname{diag}(\Sigma, P)) .
$$

where $P=\Omega^{-1}-\Omega^{-1} G \Sigma G^{\prime} \Omega^{-1}$. Indeed, a first order asymptotically equivalent Lagrange

multiplier-like estimator is obtained from the program $\hat{\lambda}=\arg \max _{\lambda \in \hat{\Lambda}_{n}(\hat{\beta})} \hat{P}_{n}^{\rho}(\hat{\beta}, \lambda)$ for any estimator $\hat{\beta}$ first order asymptotically equivalent estimator to GEL, e.g., efficient 2SGMM, MD or GMC, with associated empirical probalities consequently defined as in (3.8); see Brown and Newey (2002).

\section{TESTS}

\subsection{Over-Identifying Moment Conditions}

An important hypothesis of interest to empirical researchers is whether or not the moment conditions (2.1) hold. Consider the null hypothesis

$$
H_{0}: \mathbb{E}[g(z, \beta)]=0 \text { for some } \beta \in \mathcal{B}
$$

and the associated alternative hypothesis

$$
H_{1}: \mathbb{E}[g(z, \beta)] \neq 0 \text { for all } \beta \in \mathcal{B} .
$$

$-n \min \left[\min _{1 \leq i \leq n} \hat{\pi}_{i}, 0\right]$, deal with this problem without affecting the large sample analysis; see Antoine et al. (2007, (2.8), (2.9), p.466). Empirical probabilities were given for EL in Owen (1988), for ET by Kitamura and Stutzer (1997), for quadratic $\rho(\cdot)$ by Back and Brown (1993) and for the general case by Brown and Newey (2002). 
Hansen (1982) considers an over-identified setting where the number of moment restrictions $m$ exceeds the number of parameters $p$ and proposes what is now commonly known as the $\mathcal{J}$-statistic to test $H_{0}$ against $H_{1}$, i.e., the optimised efficient GMM criterion

$$
\mathcal{J}_{n}=n \hat{g}(\hat{\beta})^{\prime} \hat{\Omega}(\tilde{\beta})^{-1} \hat{g}(\hat{\beta})
$$

cf. (3.2). Hansen (1982) demonstrates that if the moment restrictions (2.1) hold (and thus $H_{0}$ is true) then $\mathcal{J}_{n}$ has a limiting chi-square distribution with $m-p$ degrees of freedom with consequent asymptotic $\alpha$-level critical or rejection region $\left\{\mathcal{J}_{n}>c_{m-p}^{\alpha}\right\}$ where $\mathcal{P}\left\{\chi_{m-p}^{2}>c_{m-p}^{\alpha}\right\}=\alpha$.

Although the $\mathcal{J}$-statistic is straightforward to compute, a number of simulation studies have cast doubt on whether its asymptotic properties are a useful guide to its performance in finite samples. A number of alternative test statistics based on GEL and associated criteria have also been proposed; see Imbens et al. (1998), Kitamura and Stutzer (1997), NS and Smith (1997, 2000, 2011). These statistics parallel the classical trinity of likelihood ratio, Lagrange multiplier and score statistics; namely, a likelihood ratio (LR) form of statistic

$$
\mathcal{L R}_{n}=2 n \hat{P}_{n}^{\rho}(\hat{\beta}, \hat{\lambda})
$$

a Lagrange multiplier (LM) statistic

$$
\mathcal{L} \mathcal{M}_{n}=n \hat{\lambda}^{\prime} \hat{\Omega}(\hat{\beta}) \hat{\lambda}
$$

and a score $(\mathrm{S})$ statistic

$$
\mathcal{S}_{n}=n \hat{g}(\hat{\beta})^{\prime} \hat{\Omega}(\hat{\beta})^{-1} \hat{g}(\hat{\beta})
$$

where $\hat{\beta}$ and $\hat{\lambda}$ denote GEL or first order equivalent estimators; see section 3 . All three forms of test statistic are asymptotically equivalent to the $\mathcal{J}$-statistic with a chi-square limiting distribution with $m-p$ degrees of freedom if the moment restrictions (2.1) (and $\left.H_{0}\right)$ hold. 


\subsection{Parametric Restrictions}

Owen (1990) suggested an EL-based LR-type statistic to test the simple null hypothesis $H_{0}: \beta_{0}=\beta^{0}$ against the alternative $H_{0}: \beta_{0} \neq \beta^{0}$, where $\beta^{0}$ is a known $p$-vector of constants, when $g(z, \beta)=z-\beta$ and $\beta_{0}$ denotes the population mean, i.e., $m=p$,

$$
\mathcal{L R}_{n}^{r}=2 n \hat{P}_{n}^{\rho}\left(\beta^{0}, \hat{\lambda}\left(\beta^{0}\right)\right)
$$

where $\hat{P}_{n}^{\rho}(\beta, \lambda)$ is the EL criterion $\mathcal{E} \mathcal{L}_{n}(\beta, \lambda)(3.4), \hat{\lambda}(\beta)=\arg \max _{\lambda \in \hat{\Lambda}_{n}(\beta)} \hat{P}_{n}^{\rho}(\beta, \lambda)$ and $\hat{\Lambda}_{n}(\beta)=\left\{\lambda: \lambda^{\prime} g_{i}(\beta)>-1, i=1, \ldots, n\right\}$. If $H_{0}: \beta_{0}=\beta^{0}$ is true $\mathcal{L R}_{n}^{r}$ converges in distribution to a chi-square random variable with $p$ degrees of freedom; see Owen (1990, Theorem 1, p.91). ${ }^{5}$

This framework may be straightforwardly generalised to enable tests of functions of $\beta_{0}$ to be constructed, i.e.,

$$
H_{0}: r\left(\beta_{0}\right)=0 \text { against } H_{1}: r\left(\beta_{0}\right) \neq 0
$$

where $r(\cdot)$ is a known differentiable $s$-vector of functions with dimension $s \leq p$; the moment restrictions $(2.1) \mathbb{E}\left[g\left(z, \beta_{0}\right)\right]=0$ are maintained throughout.

A number of classical-like GEL statistics have been proposed for testing $H_{0}: r\left(\beta_{0}\right)=0$ against $H_{1}: r\left(\beta_{0}\right) \neq 0$ which are GEL counterparts of those suggested for GMM in Newey and West (1987). Let $R(\beta)=\partial r(\beta) / \partial \beta^{\prime}$ with $R=R\left(\beta_{0}\right)$ of full rank $s$ and define the restricted parameter space $\mathcal{B}^{r}=\{\beta \in \mathcal{B}: r(\beta)=0\}$. The restricted GEL estimator is given by

$$
\hat{\beta}^{r}=\arg \min _{\beta \in \mathcal{B}^{r}} \sup _{\lambda \in \hat{\Lambda}_{n}(\beta)} \hat{P}_{n}^{\rho}(\beta, \lambda),
$$

and $\hat{\lambda}^{r}=\arg \max _{\lambda \in \hat{\Lambda}_{n}\left(\hat{\beta}^{r}\right)} \hat{P}_{n}^{\rho}\left(\hat{\beta}^{r}, \lambda\right)$. Let $\hat{R}, \hat{G}$ and $\hat{\Omega}$ be $H_{0}$-consistent estimates of $G$ and $\Omega$ respectively, e.g., $\hat{R}=R(\hat{\beta}), \hat{G}=\hat{G}(\hat{\beta})$ and $\hat{\Omega}=\hat{\Omega}(\hat{\beta})$. Define $\hat{\Sigma}=\left(\hat{G}^{\prime} \hat{\Omega}^{-1} \hat{G}\right)^{-1}$ and $\hat{H}=\hat{\Sigma} \hat{G}^{\prime} \hat{\Omega}^{-1}$.

\footnotetext{
${ }^{5} \mathrm{Hjört} \mathrm{et} \mathrm{al.} \mathrm{(2009)} \mathrm{allow} \mathrm{the} \mathrm{number} \mathrm{of} \mathrm{parameters} p$ to diverge and approach infinity with the sample size $n$. If the moment indicator vector $g(z, \beta)$ is uniformly bounded, then the critical region $\left\{2 n \hat{P}_{n}^{\rho}\left(\beta_{0}, \hat{\lambda}\left(\beta_{0}\right)\right)>c_{p}^{\alpha}\right\}$ is still valid for an asymptotic $\alpha$-level test provided that $p^{3} / n \rightarrow 0$; see Hjört et al. (2009, Theorem 4.1, p.1098).
} 
GEL statistics for testing $H_{0}: r\left(\beta_{0}\right)=0$ against $H_{1}: r\left(\beta_{0}\right) \neq 0$ are: LR statistic:

$$
\mathcal{L R}_{n}^{r}=2 n\left(\hat{P}_{n}^{\rho}\left(\hat{\beta}^{r}, \hat{\lambda}^{r}\right)-\hat{P}_{n}^{\rho}(\hat{\beta}, \hat{\lambda})\right)
$$

W statistic:

$$
\mathcal{W}_{n}^{r}=n r(\hat{\beta})^{\prime}\left(\hat{R} \hat{\Sigma} \hat{R}^{\prime}\right)^{-1} r(\hat{\beta})
$$

LM statistic:

$$
\mathcal{L} \mathcal{M}_{n}^{r}=n\left(\hat{\lambda}^{r}-\hat{\lambda}\right)^{\prime} \hat{\Omega}\left(\hat{\lambda}^{r}-\hat{\lambda}\right)
$$

S statistic:

$$
\mathcal{S}_{n}^{r}=n \hat{g}\left(\hat{\beta}^{r}\right)^{\prime} \hat{H}^{\prime} \hat{R}^{\prime}\left(\hat{R} \hat{\Sigma} \hat{R}^{\prime}\right)^{-1} \hat{R} \hat{H} \hat{g}\left(\hat{\beta}^{r}\right)
$$

see Smith $(1997,2000,2011)$. Under standard conditions, if $H_{0}: r\left(\beta_{0}\right)=0$ holds, all of the above statistics are asymptotically equivalent and have a limiting chi-square distribution with $s$ degrees of freedom with, e.g., for $\mathcal{L R}_{n}^{r}$ (4.3), asymptotic $\alpha$-level critical or rejection region $\left\{\mathcal{L R}_{n}^{r}>c_{s}^{\alpha}\right\}$.

\subsection{Nuisance Parameters}

In some cases the moment restrictions (2.1) may depend on nuisance parameters, such as unknown functions or parameters that are not defined explicitly by the moment restrictions but can be estimated using extraneous information. To describe this setting, the moment restrictions (2.1) are redefined as

$$
\mathbb{E}\left[g\left(z, \beta_{0}, h_{0}\right)\right]=0
$$

where as before the vector of moment indicators is known up to $\beta_{0}$ but now includes the unknown vector of functions or parameters $h_{0}$.

The following examples are taken from Hjört et al. (2009) which adopts a plug-in

approach with an estimator $\hat{h}$ (say) substituted for the unknown $h_{0}$ in the moment indicator vector. 
ExAmPLE 4.1 (Symmetry.) The interest is in testing for the symmetry of the distribution of a random variable $z$ around its median $h_{0}$. Consider a particular point $z_{0}$ in the range of $z$. Then symmetry at $z_{0}$ is expressed by the restriction

$$
F_{z}\left(z_{0}\right)=1-F_{z}\left(2 h_{0}-z_{0}\right)
$$

where $F_{z}(\cdot)$ is the distribution function of $z$. Define $\beta_{0}=F_{z}\left(z_{0}\right)$. Then the moment restrictions

$$
\begin{aligned}
& \mathbb{E}\left[I\left(z \leq z_{0}\right)-\beta_{0}\right]=0 \\
& \mathbb{E}\left[I\left(z>2 h_{0}-z_{0}\right)-\beta_{0}\right]=0
\end{aligned}
$$

are equivalent to symmetry at $z_{0}$ as defined above. The unknown population parameter $h_{0}$ is estimated by the sample median of $z$.

ExAmPLE 4.2 (Non-Parametric Regression Error Distribution.) Let $z=(y, x)^{\prime}$ where $y$ and $x$ are scalar random variables. Consider the non-parametric regression model

$$
y=h_{0}(x)+u
$$

where $h_{0}(\cdot)$ is the unknown conditional mean function $\mathbb{E}[y \mid x]$ of $y$ given $x$; the covariate $x$ and the regression error $u$ are assumed to be independent. Let $F_{u}(\cdot)$ denote the unknown distribution function of the regression error $u$. Given a fixed $z_{0}$ interest concerns the distribution function of $u$ at $z_{0}$, i.e., $\beta_{0}=F_{u}\left(z_{0}\right)$. The associated moment condition is then given by

$$
\mathbb{E}\left[I\left(y-h_{0}(x) \leq z_{0}\right)-\beta_{0}\right]=0
$$

A standard estimator for the unknown conditional mean function $h_{0}(\cdot)$ is the NadarayaWatson non-parametric estimator

$$
\hat{h}(x)=\sum_{i=1}^{n} w_{i} y_{i}
$$

where $w_{i}=\mathcal{K}_{i} / \sum_{j=1}^{n} \mathcal{K}_{j}$, where $\mathcal{K}_{i}=\mathcal{K}\left(\frac{x-x_{i}}{b_{n}}\right),(i=1, \ldots, n), k(\cdot)$ is a symmetric positive kernel function and $b_{n}$ a bandwidth parameter. 
Hjört et al. (2009) study the EL-based criterion function statistic $2 n \hat{P}_{n}^{\rho}\left(\beta^{0}, \hat{\lambda}\left(\beta^{0}\right)\right)$ (4.2) for the hypothesis $H_{0}: \beta_{0}=\beta^{0}$ based on the moment indicator vector $g(z, \beta, \hat{h})$. The limiting distribution of the EL-based statistic is non-standard for the above and other problems but can be approximated using bootstrap methods.

\section{COMPUTATION}

Because of the GEL objective function may be highly nonlinear after profiling out the auxiliary parameter vector $\lambda$ there may be severe difficulties associated with the computation of the GEL estimator of $\beta_{0}$.

Imbens and Spady (2002), Mittelhammer et al. (2005) and Kitamura (2007) advocate the following computational method. The profile GEL criterion function is defined by

$$
\begin{aligned}
\hat{P}_{n}^{\rho}(\beta) & =\hat{P}_{n}^{\rho}(\beta, \hat{\lambda}(\beta)) \\
& =\max _{\lambda \in \hat{\Lambda}_{n}(\beta)} \hat{P}_{n}^{\rho}(\beta, \lambda) .
\end{aligned}
$$

Minimisation of $\hat{P}_{n}^{\rho}(\beta)$ over $\beta \in \mathcal{B}$ constitutes the outer-loop problem which may be complex because of nonlinearity. The Davidon-Fletcher-Powell [Imbens and Spady (2002)] or the Nelder-Mead simplex [Mittelhammer et al. (2005)] methods may be efficacious for the minimisation of $\hat{P}_{n}^{\rho}(\beta)$. The latter method is possibly more preferable because neither the computation of the gradient nor the Hessian of $\hat{P}_{n}^{\rho}(\beta)$ nor numerical approximations to them are required which may sometimes be problematic in practice given the dependence of $\hat{P}_{n}^{\rho}(\beta)$ on the inner loop problem.

The inner-loop problem concerns the determination of $\hat{\lambda}(\beta)$ for given $\beta \in \mathcal{B}$, i.e.,

$$
\hat{\lambda}(\beta)=\underset{\lambda \in \hat{\Lambda}_{n}(\beta)}{\arg \max } \hat{P}_{n}^{\rho}(\beta, \lambda)
$$

Computation of $\hat{\lambda}(\beta)$ is relatively simple since $\hat{P}_{n}^{\rho}(\beta, \lambda)$ is strictly concave on $\mathcal{V}$ and can easily be achieved by Newton or related methods since the first order derivative and Hessian of $\hat{P}_{n}^{\rho}(\beta, \lambda)$ are straightforwardly obtained as

$$
\frac{\partial \hat{P}_{n}^{\rho}(\beta, \lambda)}{\partial \lambda}=\sum_{i=1}^{n} \rho_{1}\left(\lambda^{\prime} g_{i}(\beta)\right) g_{i}(\beta) / n
$$


and

$$
\frac{\partial^{2} \hat{P}_{n}^{\rho}(\beta, \lambda)}{\partial \lambda \partial \lambda^{\prime}}=\sum_{i=1}^{n} \rho_{2}\left(\lambda^{\prime} g_{i}(\beta)\right) g_{i}(\beta) g_{i}(\beta)^{\prime} / n .
$$

Since $v>-1$ is required for EL where $\rho(v)=\log (1+v)$, Kitamura (2007) suggests solving (5.1) subject to the restriction $\lambda^{\prime} g_{i}(\beta) \geq-1+\delta$ for some small $\delta>0,(i=1, \ldots, n)$. An alternative, see eq. (12.3), p.235, in Owen (2001), replaces the logarithmic function by

$$
\left\{\begin{array}{cl}
\log (x) & \text { if } x \geq \xi \\
\log (\xi)-1.5+2(x / \xi)-0.5(x / \xi)^{2} & \text { if } x<\xi
\end{array},\right.
$$

which has support given by the real line for any small number $\xi>0$. For ET where $\rho(v)=-\exp (v)$, Imbens et al. (1998) use a penalty function approach that consists in solving the following problem

$$
\max _{\beta \in \mathcal{B}, \lambda} K(\beta, \lambda)-\frac{1}{2} A K_{\lambda}(\beta, \lambda)^{\prime} W^{-1} K_{\lambda}(\beta, \lambda)
$$

where $K(\beta, \lambda)=\log \left(\sum_{i=1}^{n} \exp \left(\lambda^{\prime} g_{i}(\beta)\right) / n\right), K_{\lambda}(\beta, \lambda)=\partial K(\beta, \lambda) / \partial \lambda, W$ is a positive definite matrix and $A$ a positive scalar that can take large values. Imbens et al. (1998) choose $W$ as $W=K_{\lambda \lambda}(\tilde{\beta}, \tilde{\lambda})+K_{\lambda}(\tilde{\beta}, \tilde{\lambda}) K_{\lambda}(\tilde{\beta}, \tilde{\lambda})^{\prime}$ where $K_{\lambda \lambda}(\beta, \lambda)=\partial^{2} K(\beta, \lambda) / \partial \lambda \partial \lambda^{\prime}$ and $(\tilde{\beta}, \tilde{\lambda})$ are initial estimates, e.g., the initial root- $n$ consistent estimator $\tilde{\beta}$ used in 2SGMM and $\tilde{\lambda}=\hat{\lambda}(\tilde{\beta})$ obtained from $(5.1){ }^{6}$

An additional computational issue is that GEL requires the associated empirical probabilities to be chosen so that, not only $\pi_{i} \geq 0,(i=1, \ldots, n)$, and $\sum_{i=1}^{n} \pi_{i}=1$ hold but the first order condition $\sum_{i=1}^{n} \pi_{i} g_{i}(\beta)=0$ is automatically satisfied, i.e., $0 \in$ $\left\{\sum_{i=1}^{n} \pi_{i} g_{i}(\beta) \mid \pi_{i} \geq 0,(i=1, \ldots, n), \sum_{i=1}^{n} \pi_{i}=1\right\}$ the convex hull of $\left\{g_{i}(\beta)\right\}_{i=1}^{n}$. In finite samples, this may not be possible for particular data configurations $\left\{g_{i}(\beta)\right\}_{i=1}^{n}$. An attractive solution, adjusted EL, proposed recently in Chen et al. (2008) and Liu and Chen (2010) is to add a new data point to $\left\{g_{i}(\beta)\right\}_{i=1}^{n}$ defined by $g_{n+1}(\beta)=-a_{n} \hat{g}(\beta)$, where $\left\{a_{n}\right\}$ denotes a positive sequence, that thereby guarantees that 0 is in the convex hull of $g_{i}(\beta),(i=1, \ldots, n+1)$, since $\hat{g}(\beta)$ and $g_{n+1}(\beta)$ lie in this set and have opposite sign. In addition, the non-negativity of the empirical probabilities $\hat{\pi}_{i},(i=1, \ldots, n)$,

\footnotetext{
${ }^{6}$ Chaussé (2010) discusses computation of GMM and GEL using R. Stata code for EL is provided by Y. Kitamura at http://kitamura.sites.yale.edu/.
} 
(3.8) may not hold without explicitly imposing this condition although for large samples $\hat{\pi}_{i} \geq 0,(i=1, \ldots, n)$, with probability close to 1 if the moment restrictions $(2.1)$ are valid; footnote 4 above suggests another approach.

\section{HIGHER ORDER PROPERTIES}

\subsection{Asymptotic Bias}

NS investigated the asymptotic bias of efficient 2SGMM and GEL using the stochastic expansion

$$
\sqrt{n}\left(\hat{\beta}-\beta_{0}\right)=\psi_{n}+Q_{1, n} / \sqrt{n}+Q_{2, n} / n+Q_{3, n} / n^{3 / 2}
$$

where the quantities $Q_{j, n},(j=1, \ldots, 3)$, are random vectors that are all bounded in probability and $\psi_{n}$ has zero mean converging in distribution to a $N(0, \Sigma)$ distributed random vector.

The asymptotic bias of 2SGMM and GEL to order $O\left(n^{-1}\right)$ only requires the analysis of the behaviour of the order $1 / \sqrt{n}$ term, i.e., $Q_{1, n}$, since $\mathbb{E}\left[\psi_{n}\right]=0$ and to this order $Q_{2, n}$ also has mean zero. More precisely, the asymptotic bias of 2SGMM and GEL is defined as abias $[\hat{\beta}]=\mathbb{E}\left[Q_{1, n}\right] / n$. In general, the $O\left(n^{-1}\right)$ bias of 2 SGMM and GEL may be decomposed into four terms; for efficient 2SGMM,

$$
\operatorname{abias}[\hat{\beta}]=B_{I}+B_{G}+B_{\Omega}+B_{W}
$$

for GEL,

$$
\operatorname{abias}[\hat{\beta}]=B_{I}+\left(1+\rho_{3} / 2\right) B_{\Omega} .
$$

Each term in (6.1) and (6.2) has an interpretation. The first term $B_{I}$ is the asymptotic bias of an efficient GMM estimator based on the infeasible optimal combination of mo-

ment condition indicators $G^{\prime} \Omega^{-1} g(z, \beta)$ with first order conditions $G^{\prime} \Omega^{-1} \hat{g}(\beta)=0$. The second term $B_{G}$ arises due to the (implicit) estimation of the population Jacobian matrix $G$ whereas the estimation of the moment variance matrix $\Omega$ produces $B_{\Omega}$. The final term 
$B_{W}$ appears because of the use of the preliminary consistent estimator $\tilde{\beta}$ for $\beta_{0}$ in efficient 2SGMM and is, thus, absent for GEL. ${ }^{7}$

NS shows that not only is the term $B_{W}$ absent for GEL but the Jacobian contribution $B_{G}$ also vanishes. Clearly, if the third order derivative $\rho_{3}=-2$, the moment variance term $B_{\Omega}$ disappears from (6.2). Indeed, EL satisfies this condition. ${ }^{8}$ To illustrate these results NS, section 4.1, pp.229-230, consider a model defined through the conditional moment restriction $\mathbb{E}\left[u\left(z, \beta_{0}\right) \mid x\right]=0$ where $u\left(z, \beta_{0}\right)$ is a scalar function; estimation of $\beta_{0}$ uses the unconditional moment indicators $g\left(z, \beta_{0}\right)=q(x) \times u\left(z, \beta_{0}\right)$ with the unconditional moment restrictions $\mathbb{E}\left[g\left(z, \beta_{0}\right)\right]=0$ of $(2.1)$ where $q(\cdot)$ is a $m$-vector of functions. Interestingly, in contradistinction to 2SGMM, EL asymptotic bias does not increase with the number of moment conditions $m$.

Schennach (2007) reconsiders exponentially tilted EL EL(ET) which incorporates the ET empirical probabilities into the EL objective function and was originally proposed in Jing and Wood (1996) and Corcoran (1998) for the population mean case. EL(ET) has the same asymptotic bias as EL, is also higher order efficient and possesses desirable properties when the moment conditions (2.1) are misspecified. An alternative approach is to embed the GEL rather than ET empirical probabilities, viz. $\hat{\pi}_{i}(\beta)=$ $\rho_{1}\left(\hat{\lambda}(\beta)^{\prime} g_{i}(\beta)\right) / \sum_{j=1}^{n} \rho_{1}\left(\hat{\lambda}(\beta)^{\prime} g_{j}(\beta)\right),(i=1, \ldots, n)$, where $\hat{\lambda}(\beta)=\arg \max _{\lambda \in \hat{\Lambda}_{n}(\beta)} \hat{P}_{n}^{\rho}(\beta, \lambda)$, into the EL objective function yielding the EL(GEL) estimator

$$
\hat{\beta}=\arg \max _{\beta \in \mathcal{B}} \sum_{i=1}^{n} \log \hat{\pi}_{i}(\beta) / n ;
$$

see Smith $(2007 \mathrm{c})$. Note that the empirical probabilities $\hat{\pi}_{i}(\beta),(i=1, \ldots, n)$, must be positive, a property satisfied by, e.g., members of the Cressie-Read family with $\tau \leq 0$. EL(GEL) has the same asymptotic bias as EL(ET) and, thus, EL, but whether biascorrected EL(GEL) is also higher order efficient remains to be proven..

\footnotetext{
${ }^{7}$ Let $a$ be the $m \times 1$ vector such that $a_{j}=\operatorname{tr}\left(\Sigma \mathbb{E}\left[\partial^{2} g_{i j}\left(\beta_{0}\right) / \partial \beta \partial \beta^{\prime}\right]\right) / 2,(j=1, \ldots, m)$, where $g_{i j}(\beta)$ denotes the $j$ th element of $g_{i}(\beta), g_{i}=g_{i}\left(\beta_{0}\right)$ and $G_{i}=G_{i}\left(\beta_{0}\right)$. Also let $H_{W}=\left(G^{\prime} W^{-1} G\right)^{-1} G^{\prime} W^{-1}, H=$ $\Sigma G^{\prime} \Omega^{-1}, \bar{\Omega}_{\beta_{j}}=\mathbb{E}\left[\partial\left(g_{i}\left(\beta_{0}\right) g_{i}\left(\beta_{0}\right)^{\prime}\right) / \partial \beta_{j}\right]$ and $e_{j}$ the $j$ th unit vector. Then $B_{I}=H\left(-a+\mathbb{E}\left[G_{i} H g_{i}\right]\right) / n$, $B_{G}=-\Sigma \mathbb{E}\left[G_{i}^{\prime} P g_{i}\right] / n, B_{\Omega}=H \mathbb{E}\left[g_{i} g_{i}^{\prime} P g_{i}\right] / n$ and $B_{W}=-H \sum_{j=1}^{p} \bar{\Omega}_{\beta_{j}}\left(H_{W}-H\right)^{\prime} e_{j} / n$.

${ }^{8}$ Bias-corrected EL is higher-order efficient in the sense that it has least higher order asymptotic variance; see Theorem 6.1, p.234, in NS. Moreover, any bias-corrected GEL estimator with the same derivatives $\rho_{j}$ up to order 4 as EL, in particular, $\rho_{3}=-2$ and $\rho_{4}=-6$, shares this property.
} 
To alleviate the potential computational difficulties associated with the GEL class of estimators, see section 5, Fan et al. (2011) introduced an iterative scheme that yields estimators with the same asymptotic bias as GEL, which may be regarded as a development for the moment condition context of the classical likelihood approach in Robinson (1988a). Define the scalar function $k(v)=\left(\rho_{1}(v)+1\right) / v$, where $v \neq 0$, and $k(0)=-1$; cf. Theorem 2.3, p.224, in NS. Let $\hat{\beta}^{0}$ denote any root- $n$ consistent initial estimator of $\beta_{0}$, e.g., GMM with $\hat{W}=I_{m}$, with, for $j>0, \hat{\beta}^{(j)}$ the $j$ th iterate. Also let $\hat{\lambda}^{(j)}=\arg \max _{\lambda \in \hat{\Lambda}_{n}\left(\hat{\beta}^{(j)}\right)} \hat{P}_{n}^{\rho}\left(\hat{\beta}^{(j)}, \lambda\right)$. Define $\hat{\pi}_{i}^{(j)}=\hat{\pi}_{i}\left(\hat{\beta}^{(j)}\right), \hat{g}_{i}^{(j)}=g_{i}\left(\hat{\beta}^{(j)}\right)$, $(i=1, \ldots, n), \hat{g}^{(j)}=\hat{g}\left(\hat{\beta}^{(j)}\right), \hat{G}^{(j)}=\hat{G}\left(\hat{\beta}^{(j)}\right), \tilde{G}^{(j)}=\sum_{i=1}^{n} \hat{\pi}_{i}^{(j)} G_{i}\left(\hat{\beta}^{(j)}\right), \hat{\Omega}^{(j)}=\hat{\Omega}\left(\hat{\beta}^{(j)}\right)$ and $\tilde{\Omega}^{(j)}=\sum_{i=1}^{n} \hat{k}_{i}^{(j)} g_{i}\left(\hat{\beta}^{(j)}\right) g_{i}\left(\hat{\beta}^{(j)}\right)^{\prime}$ where $\hat{k}_{i}^{(j)}=k\left(\hat{\lambda}^{(j) \prime} \hat{g}_{i}^{(j)}\right) / \sum_{i=1}^{n} k\left(\hat{\lambda}^{(j) \prime} \hat{g}_{i}^{(j)}\right), \quad(i=1, \ldots, n)$. Then the $j$ th iterate $\hat{\beta}^{(j)}$ is defined as the solution to

$$
\hat{G}^{(j) \prime}\left[\hat{\Omega}^{(j-1)}\right]^{-1} \hat{g}^{(j)}=\left(\hat{G}^{(j-1) \prime}\left[\hat{\Omega}^{(j-1)}\right]^{-1}-\tilde{G}^{(j-1)^{\prime}}\left[\tilde{\Omega}^{(j-1)}\right]^{-1}\right) \hat{g}^{(j-1)},
$$

which may be interpreted as the recentred 2SGMM first order conditions with weight matrix $\hat{\Omega}^{(j-1)}$. Fan et al. (2011, Theorems 4.1 and 4.2, p.272) shows that, for $j>$ $0, \hat{\beta}^{(j)}$ is asymptotically equivalent to the corresponding GEL estimator and thereby asymptotically efficient. If $\hat{\beta}^{(0)}$ is itself asymptotically efficient, then $\hat{\beta}^{(j)}$ has the same asymptotic bias as GEL for $j>0$.

\subsection{Bartlett Correction}

The asymptotic distributions of the test statistics described in section 4 are approximately chi-square for sufficiently large sample sizes. As is widely recognised, the distribution of a statistic for the sample sizes typically available in practice may differ substantially from that predicted by large sample theory.

For fully parametric problems addressed by classical likelihood theory, a simple scale transformation of the (log) likelihood ratio statistic, known as a Bartlett correction, results in an improved accuracy of the asymptotic chi-square distribution theory for the finite sample behaviour of the transformed statistic; see, inter alia, Bartlett (1937) and 
Cribari-Neto and Cordeiro (1996). ${ }^{9}$ Similar results for the moment condition context are scarce; see, e.g., Owen (2001, pp. 249-251).

DiCiccio et al. (1991) discusses the smooth function model where the moment indicator vector takes the form $g(z, \beta)=z-\beta$ where $\beta_{0}$ is the population mean and considers the null hypothesis $H_{0}: \theta\left(\beta_{0}\right)=\theta^{0}$ against the alternative $H_{1}: \theta\left(\beta_{0}\right) \neq \theta^{0}$ where $\theta^{0}$ is a known $s$-vector of constants and $\theta(\cdot)$ is a $s$-vector of smooth functions of the population mean $\beta_{0}$ such that $s \leq p=m$; i.e., $r\left(\beta_{0}\right)=\theta\left(\beta_{0}\right)-\theta^{0}$, see section 4.2. DiCiccio et al. (1991) shows that the EL version of the criterion-based statistic $\mathcal{L R}_{n}^{r}=2 n\left(\hat{P}_{n}^{\rho}\left(\hat{\beta}^{r}, \hat{\lambda}^{r}\right)-\hat{P}_{n}^{\rho}(\hat{\beta}, \hat{\lambda})\right)(4.3)$ is Bartlett correctable. ${ }^{10}$ For the same set-up Baggerly (1998) proves that EL only is Bartlett correctable in the CR class of criteria. Chen and Cui (2007) provides the generalisation to the over-identified moment condition setting $\mathbb{E}[g(z, \beta)]=0(2.1)$ where $m \geq p$ dealing with the respective null and alternative hypotheses $H_{0}: \beta_{0}=\beta^{0}$ and $H_{1}: \beta_{0} \neq \beta^{0}$ demonstrating that the EL criterion statistic (4.3) is also Bartlett correctable in this case. ${ }^{11}$ If interest solely concerns the null hypothesis $H_{0}: \beta_{10}=\beta_{1}^{0}$ expressed in terms of a sub-vector of $\beta=\left(\beta_{1}^{\prime}, \beta_{2}^{\prime}\right)^{\prime}$, Chen and Cui (2006) shows that, if the nuisance parameter vector $\beta_{2}$ is first profiled out from the EL criterion, then the resultant EL statistic for $H_{0}: \beta_{10}=\beta_{1}^{0}$ against $H_{1}: \beta_{10} \neq \beta_{1}^{0}$ is Bartlett correctable.

Matsushita and Otsu (2013) prove that the EL criterion function statistic (4.1) for over-identifying moment conditions is Bartlett correctable, see section 4.1. Moreover, the adjusted EL criterion statistic [Chen et al. (2008) and Liu and Chen (2010)], see section 5, for testing over-identifying moments achieves the same accuracy as the Bartlett corrected EL criterion statistic if the adjustment factor $a_{n}$ is chosen such that $a_{n}=B_{c} / 2$ where $B_{c}$ is the Bartlett correction.

\footnotetext{
${ }^{9}$ Briefly the Bartlett correction of the classical likelihood ratio statistic $\mathcal{L} \mathcal{R}$ takes the form $\mathcal{L} \mathcal{R}_{c}=$ $\mathcal{L R} /\left(1+B_{c} / n\right)$ where the factor $B_{c}$ is a function of moments of derivatives of the log-likelihood function that typically can be consistently estimated.

${ }^{10}$ Jing and Wood (1996) demonstrates that the EL(ET) criterion test statistic is not Bartlett correctable.

${ }^{11}$ Liu and Chen (2010) shows that the test based on the adjusted EL criterion, see section 5, achieves the same accuracy as the Bartlett corrected EL statistic if the adjustment factor is set as $a_{n}=B_{c} / 2$, where $B_{c}$ denotes the Bartlett correction.
} 


\subsection{Large Deviations}

Consider two distinct simple hypotheses $H_{0}: \beta_{0}=\beta^{0}$ and $H_{1}: \beta_{0}=\beta^{1}$ where $\beta^{0}$ and $\beta^{1}$ are known $p$-vectors of constants and $\beta^{0} \neq \beta^{1}$. Let $\delta_{0}$ and $\delta_{1}$ denote the respective probabilities of Type I and Type II errors. Ideally these probabilities should be set as low as possible. Since these probabilities are inversely related Neyman-Pearson theory advises minimising $\delta_{1}$ (or equivalently maximising power $1-\delta_{1}$ ) for fixed $\delta_{0}$. As is wellknown by the Neyman-Pearson Lemma for given size $\delta_{0}$ the LR test of $H_{0}: \beta_{0}=\beta^{0}$ against $H_{1}: \beta_{0}=\beta^{1}$ is the most powerful.

Hoeffding (1965) considered a similar setting but where the probabilities of both Type I and Type II error probabilities, $\delta_{0 n}$ and $\delta_{1 n}$ respectively, depend on the sample size $n$ and approach zero exponentially as $n$ increases. Thus the Type I and II errors correspond to extreme tail events for sufficiently large $n$. Hoeffding (1965) showed the large deviation result that among those tests of $H_{0}: \beta_{0}=\beta^{0}$ against $H_{1}: \beta_{0}=\beta^{1}$ that satisfy

$$
\lim \sup _{n \rightarrow \infty} n^{-1} \log \delta_{0 n} \leq-\eta
$$

for $\eta$ fixed, the LR test minimises

$$
\lim _{n \rightarrow \infty} n^{-1} \log \delta_{1 n}
$$

i.e., the LR test minimizes the probability of a Type II error.

Kitamura et al. (2012) applied the large deviation theory of Hoeffding (1965) to tests of over-identifying moment restrictions, see section 4.1. In general there is no test that satisfies (6.3). However, if certain probability distributions that satisfy the moment restrictions (2.1) are eliminated then the following results hold: (a) the test of over-identifying moment restrictions $H_{0}: \mathbb{E}[g(z, \beta)]=0$ for some $\beta \in \mathcal{B}$ formed from

the EL-based criterion $\mathcal{L R}_{n}=2 n \hat{P}_{n}^{\rho}(\hat{\beta}, \hat{\lambda})(4.1)$ satisfies $(6.3)$; (b) among all tests that satisfy (6.3), the EL-based criterion test minimises (6.4) if the alternative hypothesis $H_{1}: \mathbb{E}[g(z, \beta)] \neq 0$ for all $\beta \in \mathcal{B}$ deviates sufficiently from $H_{0}$. 


\subsection{Robustness}

The robustness properties of the ML estimator are examined in Beran (1977). Small deviations from the assumed parametric density function can lead to large variations in the log likelihood function demonstrating a lack of robustness of ML in this sense. Beran (1977) shows that an alternative parametric estimator based on the minimisation of a discrepancy measure formulated in terms of Hellinger distance is robust but also shares the asymptotic efficiency property of ML.

Let $f_{0}(\cdot)$ denote the density function of the data observation $z$ commensurate with the moment condition $\mathbb{E}[g(z, \beta)]=0$ (2.1) satisfied at the unique value $\beta_{0}$ of $\beta \in \mathcal{B}$. Based on random samples drawn from density functions in a neighbourhood of $f_{0}(\cdot)$ Kitamura et al. (2013) analyses the robustness properties of estimators of a known scalar function $m(\cdot)$ of $\beta_{0}$ in terms of their asymptotic maximum bias and mean squared error. In the class of Fisher consistent and regular estimators, which includes GMM and GEL, maximum bias is minimised by the minimum Hellinger distance estimator (MHDE) computed using the trimmed moment indicators $g(z, \beta) I\left(\sup _{\beta \in \mathcal{B}}\|g(z, \beta)\| \leq m_{n}\right)$ where $\left\{m_{n}\right\}$ is a positive valued sequence that approaches infinity with sample size $n$. Additionally, mean squared error is also minimized by MHDE based on the moment indicator vector $g(z, \beta)$. MHDE corresponds to the $\mathrm{CR}$ discrepancy measure with parameter $\tau=-1 / 2$; thus $\mathrm{MHDE}$ is also GEL and is thereby asymptotically efficient, see section 3.

\section{CONDITIONAL MOMENTS}

Many empirical problems concern models defined through conditional rather than unconditional moment restrictions. Let $u(z, \beta)$ be a known $J$-vector of functions of the random vector of observables $z$ and the unknown $p$-vector of parameters $\beta$ which as before constitute the object of inferential interest. We consider models defined by the following conditional moments

$$
\mathbb{E}\left[u\left(z, \beta_{0}\right) \mid x\right]=0
$$

where $x$ is a $d_{x}$-dimensional subvector of $z$. 
The following examples illustrate this framework.

EXAMPLE 2.2 (Quantile Regression cont.) The quantile regression model is redefined by the conditional probability statement $\mathcal{P}\left\{y \leq x^{\prime} \beta_{0} \mid x\right\}=\theta$. The conditional moment condition (7.1) defining $\theta$-conditional quantile regression is $\mathbb{E}\left[\theta-I\left(y \leq x^{\prime} \beta_{0}\right) \mid x\right]=0$ or

$$
\mathbb{E}[\theta-I(u \leq 0) \mid x]=0
$$

where $u=y-x^{\prime} \beta_{0}$. Here $\beta_{0}$ is the unique value of $\beta$ that satisfies $\mathbb{E}\left[\theta-I\left(y \leq x^{\prime} \beta\right) \mid x\right]=0$. Similarly to section 2 , the unconditional moment restriction $\mathbb{E}[x(\theta-I(u \leq 0))]=0$ holds as does $\mathbb{E}[q(x)(\theta-I(u \leq 0))]=0$ for suitably defined vectors of functions $q(\cdot)$ of $x$.

ExAmPLE 2.3 (Instrumental Variables cont.) The conditional mean restriction $\mathbb{E}[y-$ $\left.x^{\prime} \beta_{0} \mid w\right]=0$ or $\mathbb{E}[u \mid w]=0$, cf. (7.1), where $u=y-x^{\prime} \beta_{0}$, is often assumed in a linear regression setting; see inter alia Greene (2008) and Davidson and MacKinnon (2004). Here $\beta_{0}$ is the unique value of $\beta$ that satisfies $\mathbb{E}\left[y-x^{\prime} \beta \mid w\right]=0$. Similarly to Example 2.2 above, the unconditional moment restriction $\mathbb{E}[q(w) u]=0$ is implied, where $q(\cdot)$ is a vector of functions of the instruments $w$; in particular, $\mathbb{E}[w u]=0$ holds. For standard linear regression, the conditional mean restriction $\mathbb{E}[y \mid x]=x^{\prime} \beta_{0}$ or $\mathbb{E}[u \mid x]=0$ would be the standard assumption. It is well-known, see Cragg (1983), that unless the linear regression model is conditionally homoskedastic, i.e., $\operatorname{var}[u \mid x]$ is constant or invariant to $x$, instrumental variable estimation of $\beta_{0}$ based on the unconditional moment restriction $\mathbb{E}[q(x) u]=0$ when $q(x)$ includes $x$ is more efficient that least squares.

GMM- and EL-type estimators of $\beta_{0}$ that achieve the semiparametric efficiency lower bound [Chamberlain (1987)] have been proposed in Donald, Imbens and Newey (2004) [DIN], Kitamura, Tripathi and Ahn (2004) [KTA] and Zhang and Gijbels (2003). The DIN approach uses unconditional moment restrictions based on particular classes of approximating functions such as splines and power series whereas that of KTA and Zhang and Gijbels (2003) employs kernel smoothed moment indicator functions. Otsu (2007) 
extends the conditional EL approach of KTA and Zhang and Gijbels (2003) to conditional moment restriction models that incorporate unknown infinite dimensional functions requiring nonparametric estimation, models that were studied previously in $\mathrm{Ai}$ and Chen (2003) using the method of sieves applied to GMM.

\subsection{Approximating Functions}

DIN note that any function can be approximated arbitrarily well by linear combinations of certain basis or approximating functions when the number of functions is allowed to approach infinity with sample size $n$. Particular examples of admissible classes of approximating functions are splines, power series and Fourier series; see, e.g., Powell (1981).

To be more specific, let $K$ be the number of approximating functions and define $q^{K}(x)=\left(q_{1 K}(x), \ldots, q_{K K}(x)\right)^{\prime}$ as the $K$-vector of approximating functions. The consequent vector of unconditional moment indicators is given by

$$
g^{K}(z, \beta)=u(z, \beta) \otimes q^{K}(x)
$$

cf. (2.1). Lemma 2.1, p.58, in DIN demonstrates a formal equivalence between the sequence of unconditional moment constraints $\mathbb{E}\left[g^{K}\left(z, \beta_{0}\right)\right]=0(7.2), K \rightarrow \infty$, and the conditional moment restriction $\mathbb{E}\left[u\left(z, \beta_{0}\right) \mid x\right]=0(7.1)$. More precisely, by the law of iterated expectations $\mathbb{E}\left[g^{K}\left(z, \beta_{0}\right)\right]=0$ for all $K$ if $\mathbb{E}\left[u\left(z, \beta_{0}\right) \mid x\right]=0$ and, moreover, $\mathbb{E}\left[g^{K}\left(z, \beta_{0}\right)\right] \neq 0$ for all $K$ large enough if $\mathbb{E}\left[u\left(z, \beta_{0}\right) \mid x\right] \neq 0$.

Consequently EL, GMM and GEL may be applied using the unconditional moment indicator vector $g^{K}(z, \beta)(7.2)$. DIN shows that if $K$ approaches infinity at an appropriate rate dependent on the approximating functions and the estimator employed the resultant estimators are consistent and achieve the semiparametric efficiency lower bound $\mathbb{E}\left[D(x)^{\prime} \Sigma(x)^{-1} D(x)\right]^{-1}\left[\right.$ Chamberlain (1987)], where $D(x)=\mathbb{E}\left[\partial u\left(z, \beta_{0}\right) / \partial \beta^{\prime} \mid x\right]$ and $\Sigma(x)=\mathbb{E}\left[u\left(z, \beta_{0}\right) u\left(z, \beta_{0}\right)^{\prime} \mid x\right]$. 


\subsection{Conditional (G)EL}

Let $u_{i}(\beta)=u\left(z_{i}, \beta\right),(i=1, \ldots, n)$. The KTA approach, see also Zhang and Gijbels (2003), modifies EL by scaling the standard EL criterion (3.4) using the positive weights $w_{i j},(i, j=1, \ldots, n)$, i.e.,

$$
\mathcal{C} \mathcal{E} \mathcal{L}_{n}(\beta, \lambda)=\sum_{i=1}^{n} \mathbb{T}_{i, n} \sum_{j=1}^{n} w_{i j} \log \left(1+\lambda_{i}^{\prime} u_{j}(\beta)\right) / n,
$$

where $\lambda=\left(\lambda_{1}^{\prime}, \ldots, \lambda_{n}^{\prime}\right)^{\prime}, w_{i j}=\mathcal{K}_{i j} / \sum_{k=1}^{n} \mathcal{K}_{i k}, \mathcal{K}_{i j}=\mathcal{K}\left(\frac{x_{i}-x_{j}}{b_{n}}\right), \mathcal{K}(\cdot)$ is a symmetric positive kernel and $b_{n}$ a bandwidth parameter. The trimming function $\mathbb{T}_{i, n}$ is required to ensure the denominator of the weights $w_{i j}$ is bounded away from zero, i.e., $\mathbb{T}_{i, n}=I\left(\hat{h}\left(x_{i}\right) \geq b_{n}^{\tau}\right)$ for some $\tau \in(0,1)$ where $\hat{h}(x)=\sum_{j=1}^{n} \mathcal{K}\left(\frac{x-x_{j}}{b_{n}}\right) / n b_{n}^{d_{x}}$ is the standard kernel estimator for the density $h(\cdot)$ of $x$ and $I(\cdot)$ is an indicator function. ${ }^{12}$ Note that $\mathcal{C} \mathcal{E} \mathcal{L}_{n}(\beta, \lambda)$ employs the Nadaraya-Watson estimator $\sum_{j=1}^{n} w_{i j} \log \left(1+\lambda_{i}^{\prime} u_{j}(\beta)\right)$ of the conditional expectation of $\log \left(1+\lambda_{i}^{\prime} u_{j}(\beta)\right)$ given $x_{i}$, i.e. $\mathbb{E}\left[\log \left(1+\lambda_{i}^{\prime} u_{j}(\beta)\right) \mid x_{i}\right], \quad(i=1, \ldots, n)$, and, thus, may be regarded as an estimator of the average conditional expectation $\sum_{i=1}^{n} \mathbb{E}\left[\log \left(1+\lambda_{i}^{\prime} u_{i}(\beta)\right) \mid x_{i}\right] / n$.

Let $\Lambda_{n}=\left\{\lambda \in \mathbb{R}^{J}:\|\lambda\| \leq C n^{-1 / m}\right\}$ for some finite constant $C>0 .{ }^{13}$ The conditional EL estimator is the solution to a saddle point problem

$$
\hat{\beta}=\arg \inf _{\beta \in \mathcal{B}} \sum_{i=1}^{n} \mathrm{~T}_{i, n} \sup _{\lambda_{i} \in \Lambda_{n}} \sum_{j=1}^{n} w_{i j} \log \left(1+\lambda_{i}^{\prime} u_{j}(\beta)\right) / n
$$

with the Lagrange multiplier estimator $\hat{\lambda}_{i}(\beta)$ defined by $\hat{\lambda}_{i}(\beta)=\arg \max _{\lambda_{i} \in \Lambda_{n}} \sum_{j=1}^{n} w_{i j} \ln (1+$ $\left.\lambda_{i}^{\prime} u\left(z_{j}, \beta\right)\right)$. The conditional EL estimator $\hat{\beta}(7.4)$ is consistent and achieves the semiparametric efficiency lower bound $\mathbb{E}\left[D(x)^{\prime} \Sigma(x)^{-1} D(x)\right]^{-1}$; see KTA. ${ }^{14}$

Conditional EL was subsequently generalised in Smith (2007a, b) for GEL and the Cressie-Read power divergence family with criterion $\sum_{i=1}^{n} \mathbb{T}_{i, n} \sum_{j=1}^{n} w_{i j}\left[\rho\left(\lambda_{i}^{\prime} u_{j}(\beta)\right)-\right.$

\footnotetext{
${ }^{12}$ The criterion $\mathcal{C E}_{\mathcal{L}_{n}(\beta, \lambda)}$ (7.3) is obtained from the programme $\max _{\beta,\left\{\pi_{i j}\right\}_{i, j=1}^{n}} \sum_{i=1}^{n} \mathbb{T}_{i, n} \sum_{j=1}^{n} w_{i j} \log \pi_{i j}$ subject to $\pi_{i j} \geq 0, \sum_{j=1}^{n} \pi_{i j}=1$ and $\sum_{j=1}^{n} \pi_{i j} u_{j}(\beta)=1$, $(i, j=1, \ldots, n)$. Note that $\pi_{i j}$ has the interpretation as the probability $\mathcal{P}\left\{z=z_{j} \mid x=x_{i}\right\},(i, j=1, \ldots, n)$, and $\lambda_{i}$ is the Lagrange multiplier associated with the sample moment constraint $\sum_{j=1}^{n} \pi_{i j} u_{j}(\beta)=1$, $(i=1, \ldots, n)$.

${ }^{13}$ For technical reasons $m$ is a positive integer such that $m \geq 8$.

${ }^{14}$ Tripathi and Kitamura (2003) propose a test statistic based on conditional EL. See also Smith (2007a, b) for conditional GEL-based test statistics.
} 
$\rho(0)] / n$ where $\rho(\cdot)$ is defined in section 3.4. See also Antoine et al. (2007) which proposes a similar conditional estimator based on CUE.

\subsection{Unknown Functions}

Consider the generalised form of the vector of conditional moment restrictions $\mathbb{E}[u(z, \beta) \mid x]=$ 0 (7.1) given by

$$
\mathbb{E}\left[u\left(z, \beta_{0}, h_{0}\left(x_{z}\right)\right) \mid x\right]=0 .
$$

Here, as above, $u\left(z, \beta_{0}, h_{0}\left(x_{z}\right)\right)$ is a $J$-vector of known functions but now includes the unknown vector $h_{0}(\cdot)$ of smooth functions of the subvector $x_{z}$ of the conditioning variables $x$ as an argument. Although $\beta_{0}$ remains of central inferential interest, the unknown function $h_{0}(\cdot)$ is of interest too. Examples of this general framework include partially linear regression $u\left(z, \beta_{0}, h_{0}\left(x_{z}\right)\right)=y-x_{1}^{\prime} \beta_{0}-h_{0}\left(x_{z}\right)$ where $x=\left(x_{1}^{\prime}, x_{z}^{\prime}\right)^{\prime}$ [Robinson (1988b)] and single index regression $u\left(z, \beta_{0}, h_{0}\left(x_{z}\right)\right)=y-h_{0}\left(x_{z}^{\prime} \beta_{0}\right)$ where $x=x_{z}$ [Powell et al. (1989) and Ichimura (1993)].

Let the true parameter vector $\alpha_{0}=\left(\beta_{0}^{\prime}, h_{0}^{\prime}\right)^{\prime}$ with parameter space $\mathcal{A}=\mathcal{B} \times \mathcal{H}$. Consequently the conditional moment restriction (7.5) may be rewritten as $\mathbb{E}\left[u\left(z, \alpha_{0}\right) \mid x\right]=0$. Although with this redefinition the conditional moment restriction (7.5) now superficially resembles (7.1) clearly the KTA conditional EL estimator cannot be applied without modification since $\alpha_{0}$ contains the infinite dimensional parameter $h_{0}(\cdot)$.

Let $u_{i}(\alpha)=u\left(z_{i}, \alpha\right),(i=1, \ldots, n)$. The penalized EL criterion proposed in Otsu (2007) adopts the approach of Shen (1997) modifying the conditional EL criterion $\mathcal{C} \mathcal{E} \mathcal{L}_{n}(\beta, \lambda)$ (7.3) as

$$
\mathcal{P} \mathcal{E} \mathcal{L}_{n}(\alpha, \lambda)=\sum_{i=1}^{n} \mathbb{T}_{i, n} \sum_{j=1}^{n} w_{i j} \log \left(1+\lambda^{\prime} u_{j}(\alpha)\right) / n-\phi_{n} \mathcal{J}(h)
$$

with the incorporation of the penalty function $\mathcal{J}(\cdot)$ in order to impose some restrictions on the parameter space $\mathcal{A}$; the positive valued sequence $\left\{\phi_{n}\right\}$ of penalisation constants is chosen so as to converge to zero with sample size $n$ at rate $o\left(n^{-1 / 2}\right)$. Examples of penalty functions $\mathcal{J}(\cdot)$ may be found in section 3 of Shen (1997), e.g., to impose twice 
differentiability on the resultant estimator of $h_{0}$. The penalised EL estimator $\hat{\alpha}$ is the solution to a saddle point problem

$$
\hat{\alpha}=\arg \inf _{\alpha \in \mathcal{A}} \sum_{i=1}^{n} \mathbb{T}_{i, n} \sup _{\lambda_{i} \in \mathbb{R}^{J}} \sum_{j=1}^{n} w_{i j} \log \left(1+\lambda_{i}^{\prime} u_{j}(\beta)\right) / n
$$

with the Lagrange multiplier estimator $\hat{\lambda}_{i}(\beta)$ defined by $\hat{\lambda}_{i}(\beta)=\arg \max _{\lambda_{i} \in \mathbb{R}^{J}} \sum_{j=1}^{n} w_{i j} \log (1+$ $\left.\lambda_{i}^{\prime} u\left(z_{j}, \beta\right)\right)$. Otsu (2007) proves the consistency of the penalised EL estimator $\hat{\alpha}=\left(\hat{\beta}^{\prime}, \hat{h}^{\prime}\right)^{\prime}$ for $\alpha_{0}=\left(\beta_{0}^{\prime}, h_{0}^{\prime}\right)^{\prime}$ together with the respective convergence rates of $\hat{\beta}$ and $\hat{h}$. Moreover, the penalised EL estimator $\hat{\beta}$ (7.6) of $\beta_{0}$ is asymptotically normal and achieves the semi-parametric efficiency lower bound $\mathbb{E}\left[D(x)^{\prime} \Sigma(x)^{-1} D(x)\right]^{-1}$, where now $D(x)=$ $\mathbb{E}\left[\partial u\left(z, \alpha_{0}\right) / \partial \beta^{\prime} \mid x\right]$ and $\Sigma(x)=\mathbb{E}\left[u\left(z, \alpha_{0}\right) u\left(z, \alpha_{0}\right)^{\prime} \mid x\right] .$.

In an earlier paper $\mathrm{Ai}$ and Chen (2003) suggest a sieve minimum distance approach similar to GMM in which the conditional moment indicator vector $u_{i}(\cdot)$ is estimated using sieves rather than kernel functions and the unknown functions comprising $h_{0}$ are also likewise approximated. Unlike Otsu (2007), Ai and Chen (2003) assume $\mathcal{A}$ is compact which has the advantage of allowing the unknown vector of functions $h_{0}$ to depend on $z$ rather than solely a subvector of $x$ thus permitting the inclusion of endogenous variables. More recently, for a similar set-up permitting endogenous components of the unknown function vector $h_{0}$, Otsu (2011) applies the KTA conditional EL method with $h_{0}$ approximated by sieves as in Ai and Chen (2003). Chen and Pouzo (2009) generalises the sieve minimum distance method of $\mathrm{Ai}$ and Chen (2003) to allow for non-smooth functions to comprise $h_{0}$ together with a bootstrap procedure for improved inference. ${ }^{15}$ All of these methods result in consistent and asymptotically equivalent normally distributed estimators of $\beta_{0}$ that achieve the semiparametric efficiency lower bound $\mathbb{E}\left[D(x)^{\prime} \Sigma(x)^{-1} D(x)\right]^{-1}$.

\footnotetext{
${ }^{15}$ For stationary and ergodic data Chen and Pouzo (2012) establishes convergence rates for a penalised sieve minimum distance estimator of $h_{0}$ in circumstances that likewise permit non-smooth unknown functions and the possible inclusion of endogenous variables. The penalisation, cf. Otsu (2007), avoids the necessity of restricting the parameter space $\mathcal{A}$ to be compact and may ease computational difficulties associated with the sieve minimum distance methods of Ai and Chen (2003) and Chen and Pouzo (2009).
} 


\section{WEAKLY DEPENDENT DATA}

In this section, $z_{t},(t=1, \ldots, T)$, denotes $T$ observations on a finite dimensional stationary and strongly mixing process $\left\{z_{t}\right\}_{t=1}^{\infty}$. The moment indicator vector $g\left(z_{t}, \beta\right)$ is defined as previously as an $m$-vector of known functions of the data observation $z_{t}$ and the $p$-vector $\beta$ of unknown parameters which are the object of inferential interest, where $m \geq p$. It is assumed that the true parameter vector $\beta_{0}$ uniquely satisfies the moment condition

$$
\mathbb{E}\left[g\left(z_{t}, \beta\right)\right]=0
$$

where $\mathbb{E}[\cdot]$ denotes expectation taken with respect to the unknown distribution of $z_{t}$. Since (8.1) may arise in many cases from conditional moment restrictions, $z_{t}$ may also include lagged endogenous and current and lagged values of exogenous variables.

Define $g_{t}(\beta)=g\left(z_{t}, \beta\right),(t=1, \ldots, T)$, and $\hat{g}(\beta)=T^{-1} \sum_{t=1}^{T} g_{t}(\beta)$. Let $k(\cdot)$ denote a kernel function that satisfies the mild regularity conditions stated in Smith (2011) and define $k_{j}=\int_{-\infty}^{\infty} k(a)^{j} d a, j=1,2,3$, with $k=k_{1} / k_{2}$. The bandwidth parameter $S_{T}$ diverges to infinity at an appropriate rate dependent on the kernel function $k(\cdot)$ and sample size $T$.

\subsection{Efficient $(\mathrm{G}) \mathrm{EL}$}

Kitamura and Stutzer (1997) [KS] observed that applying standard ET to the moment indicators $\left\{g_{t}(\beta)\right\}_{t=1}^{T}$ results in a consistent but asymptotically inefficient estimator of $\beta_{0}$ if there is dependence. To deal with this problem KS modifies the ET criterion by basing it on a smoothed version of the moment indicators $\left\{g_{t}(\beta)\right\}_{t=1}^{T}$ obtained using the truncated or uniform kernel function. Smith (1997, 2011) discusses GEL employing general kernel functions. For suitable choices of the kernel function $k(\cdot)$ GEL is asymptotically efficient. ${ }^{16}$ An alternative approach is suggested in Kitamura (1997) using blockwise EL. The exposition that follows is based on the approach in Smith (2011).

\footnotetext{
${ }^{16}$ In general, the first order condition for the GEL estimator $\tilde{\beta}$ using the unsmoothed moment indicators $\left\{g_{t}(\beta)\right\}_{t=1}^{T}$ may be expressed as $\left[\sum_{t=1}^{T} \tilde{\pi}_{t} G_{t}(\tilde{\beta})\right]^{\prime}\left[\sum_{t=1}^{T} \tilde{p}_{t} g_{t}(\tilde{\beta}) g_{t}(\tilde{\beta})^{\prime}\right]^{-1} \hat{g}(\tilde{\beta})=0$ where $G_{t}(\beta)=\partial g_{t}(\beta) / \partial \beta^{\prime}, \quad \tilde{\pi}_{t}=\rho_{1}\left(\tilde{\lambda}^{\prime} g_{t}(\tilde{\beta})\right) / \sum_{s=1}^{T} \rho_{1}\left(\hat{\lambda}^{\prime} g_{s}(\tilde{\beta})\right), \tilde{p}_{t}=p\left(\tilde{\lambda}^{\prime} g_{t}(\tilde{\beta})\right) / \sum_{s=1}^{T} p\left(\tilde{\lambda}^{\prime} g_{s}(\tilde{\beta})\right), \quad(t=$ $1, \ldots, T)$, with the function $p(\cdot)$ defined as $p(v)=\left[\rho_{1}(v)+1\right] / v, v \neq 0$ and $p(0)=-1$, and $\tilde{\lambda}=\sup _{\lambda \in \Lambda_{T}} \sum_{t=1}^{T}\left[\rho\left(\lambda^{\prime} g_{t}(\tilde{\beta})\right)-\rho(0)\right] / T$, cf. $\quad\left(3.7\right.$, with $\Lambda_{T}$ defined below. Although $\sum_{t=1}^{T} \tilde{\pi}_{t} G_{t}(\tilde{\beta})$
} 
Define the smoothed moment indicators

$$
g_{t T}(\beta)=\frac{1}{S_{T}} \sum_{s=t-T}^{t-1} k\left(\frac{s}{S_{T}}\right) g_{t-s}(\beta),(t=1, \ldots, T),
$$

Examples of admissible kernel functions $k(\cdot)$ are the truncated or uniform kernel $k_{T R}(x)=$ $I(|x| \leq 1)$ and the Bartlett kernel $k_{B T}(x)=(1-|x|) I(|x| \leq 1)$.

GEL criteria appropriate for weakly dependent data are defined by

$$
\hat{P}_{T}^{\rho}(\beta, \lambda)=\sum_{t=1}^{T}\left[\rho\left(k \lambda^{\prime} g_{t T}(\beta)\right)-\rho(0)\right] / T
$$

cf. section 3.4, with the GEL estimator then given by

$$
\hat{\beta}=\arg \min _{\beta \in \mathcal{B}} \sup _{\lambda \in \Lambda_{T}} \hat{P}_{T}^{\rho}(\beta, \lambda),
$$

where $\Lambda_{T}=\left\{\lambda:\|\lambda\| \leq C_{T}\right\}$ where $C_{T}$ is a positive sequence that depends on $T$ and converges to zero at an appropriate rate, see Assumption 2.4(b), p.1200, in Smith (2011). Let $\hat{\lambda}(\beta)=\arg \sup _{\lambda \in \Lambda_{T}} \hat{P}_{T}^{\rho}(\beta, \lambda)$ with $\hat{\lambda}=\hat{\lambda}(\hat{\beta})$.

Define $\Sigma=\left(G^{\prime} \Omega^{-1} G\right)^{-1}$ and $P=\Omega^{-1}-\Omega^{-1} G \Sigma G^{\prime} \Omega^{-1}$. Then, under standard regularity conditions,

$$
T^{1 / 2}\left(\hat{\beta}-\beta_{0}\right) \stackrel{d}{\rightarrow} N(0, \Sigma),\left(T / S_{T}^{2}\right)^{1 / 2} \hat{\lambda} \stackrel{d}{\rightarrow} N(0, P)
$$

and the GEL estimator $\hat{\beta}$ and the auxiliary parameter estimator $\hat{\lambda}$ are asymptotically uncorrelated. Consequently GEL is asymptotically equivalent to asymptotically efficient GMM.

\subsection{Higher Order Properties}

The literature on the higher order properties of $(\mathrm{G}) \mathrm{EL}$ for time series data is relatively limited. Kitamura (1997) shows the Bartlett correctability of the blockwise EL criterion statistic in the time series context for smooth functions of the mean; cf. section 6.2. Anatolyev (2005) investigates the asymptotic bias of 2SGMM and GEL estimators based on the smoothed moment indicators (8.2).

is a consistent estimator for $G, \sum_{t=1}^{T} \tilde{p}_{t} g_{t}(\tilde{\beta}) g_{t}(\tilde{\beta})^{\prime}$ consistently estimates the short run variance matrix $\mathbb{E}\left[g_{t}\left(\beta_{0}\right) g_{t}\left(\beta_{0}\right)^{\prime}\right]$ rather than the long run variance matrix $\Omega$ required for asymptotic efficiency. 
Anatolyev (2005) considers the 2SGMM estimator with weighting matrix the inverse of the HAC consistent estimator $S_{T} \sum_{t=1}^{T} g_{t T}(\tilde{\beta}) g_{t T}(\tilde{\beta})^{\prime} / T$ [Smith (2005)] of the moment indicator vector long-run variance matrix $\Omega$, where $\tilde{\beta}$ is a preliminary root- $T$ consistent estimator of $\beta_{0}$; CUE with the same form of weighting matrix $\left[S_{T} \sum_{t=1}^{T} g_{t T}(\beta) g_{t T}(\beta)^{\prime} / T\right]^{-1}$; GEL as in section 8.1 above.

Anatolyev (2005) confines consideration to kernels $k(\cdot)$ with bounded support, i.e., non-zero on an open interval including 0 and zero elsewhere. Similarly to the discussion in section 6.1 above the asymptotic bias of GMM and GEL may be decomposed into a number of factors, each of which has the same interpretation as in section 6.1, i.e., for GMM, ${ }^{17}$

$$
\operatorname{abias}[\hat{\beta}]=B_{I}+B_{G}+B_{\Omega}+B_{W}
$$

and, for GEL,

$$
\operatorname{abias}[\hat{\beta}]=B_{I}+\left(1+\rho_{3} k_{1} k_{3} / 2 k_{2}^{2}\right) B_{\Omega} .
$$

Note that, since $\rho_{3}=0$, the CUE asymptotic bias is $B_{I}+B_{\Omega}$; cf. section 6.1 . For further details, see Anatolyev (2005, Theorem 1, p.988).

The interpretation of these asymptotic bias terms is similar to those given earlier for the cross-section setting although their mathematical expressions differ. In particular, as there, $B_{I}$ coincides with the asymptotic bias for an infeasible GMM estimator obtained from the first order conditions $G^{\prime} \Omega^{-1} \hat{g}(\beta)=0, B_{G}$ is due to the estimation of $G$ and is absent for GEL, $B_{\Omega}$ for (implicit) estimation of $\Omega$ and $B_{W}$ from the preliminary consistent estimation of $\beta_{0}$ in $2 \mathrm{SGMM}$.

Unlike cross-section data, the $B_{\Omega}$ term does not vanish for EL unless $k_{2}^{2}=k_{1} k_{3}$ which occurs for the truncated or uniform kernel considered in KS. More generally, this term is not present for GEL criteria $\rho(\cdot)$ and kernel functions $k(\cdot)$ such that $\rho_{3}=-2\left(k_{2}^{2}\right) /\left(k_{1} k_{3}\right)$. For the GEL class $\rho(v)=-(1+\tau v)^{(1+\tau) / \tau} /(1+\tau)$ equivalent to the CR power divergence

\footnotetext{
${ }^{17}$ Let $a$ be the $m \times 1$ vector such that $a_{j}=\operatorname{tr}\left(\Sigma \mathbb{E}\left[\partial^{2} g_{t}^{j}\left(\beta_{0}\right) / \partial \beta \partial \beta^{\prime}\right]\right) / 2, \quad(j=1, \ldots, m)$, where $g_{t}^{j}(\beta)$ denotes the $j$ th element of $g_{t}(\beta), g_{t}=g_{t}\left(\beta_{0}\right), G_{t}(\beta)=\partial g_{t}(\beta) / \partial \beta^{\prime}$ and $G_{t}=G_{t}\left(\beta_{0}\right)$. Also let $H_{W}=\left(G^{\prime} W^{-1} G\right)^{-1} G^{\prime} W^{-1}, H=\Sigma G^{\prime} \Omega^{-1}, \bar{\Omega}_{\beta_{j}}(u)=\mathbb{E}\left[\partial\left(g_{t}\left(\beta_{0}\right) g_{t-u}\left(\beta_{0}\right)^{\prime}\right) / \partial \beta_{j}\right]$ and $e_{j}$ the $j$ th unit vector. Then $B_{I}=H\left(-a+\sum_{u=-\infty}^{\infty} \mathbb{E}\left[G_{t} H g_{t-u}\right]\right) / T, B_{G}=-\Sigma \sum_{u=-\infty}^{\infty} \mathbb{E}\left[G_{t}^{\prime} P g_{t-u}\right] / T$, $B_{\Omega}=H \sum_{u, v=-\infty}^{\infty} \mathbb{E}\left[g_{t} g_{t-u}^{\prime} P g_{t-v}\right] / T$ and $B_{W}=-H \sum_{j=1}^{p} \sum_{u=-\infty}^{\infty} \bar{\Omega}_{\beta_{j}}(u)\left(H_{W}-H\right)^{\prime} e_{j} / T$.
} 
family the choice $\tau=\left(k_{1} k_{3}-2\left(k_{2}^{2}\right)\right) /\left(k_{1} k_{3}\right)$ is required.

\section{CONCLUSIONS}

A number of open research areas remain. For example, as noted in section 5, although (G)EL methods have attractive theoretical large sample properties their computation raises serious practical difficulties because of the induced nonlinearity arising from the necessity to solve a saddle point problem. Fast and reliable algorithms for $(\mathrm{G})$ EL when the number of moment restrictions and parameters to be estimated are large would enable (G)EL to be applied to a wider variety of empirical problems than is currently the case and allow more substantial and detailed simulation studies of these techniques to be undertaken. Bootstrap methods specifically designed for the application of $(\mathrm{G}) \mathrm{EL}$ are scarce, although a notable exception is Canay (2010). As noted in section 3, (G)EL imposes the moment restrictions in the sample. Brown and Newey (2002) exploit this feature for the cross-section setting by reweighting moment indicator observations using the empirical probabilities. Therefore, unlike that of Hall and Horowitz (1996), their bootstrap method based on the resampling of the reweighted moment indicators does not require moments to be explicitly centred. In general the higher order properties of this and other related procedures remain to be investigated.

The literature on estimation and inference for models specified by moment condition constraints is vast. Hence, because of space limitations, this review can only be partial in terms of its coverage. A number of other important areas are also currently exciting considerable research effort.

This review has concentrated on models in which parameters are point identified. Moment condition models in which parameters are only set or partially identified have received a great deal of attention in the recent literature; see, e.g., Andrews and Shi (2013), which considers moment inequality restrictions, and the references therein.

The extensive literature on weak identification, in particular, weak instruments in the regression context, was initiated by the study in Angrist and Krueger (1991) of the returns 
to education. Weak identification essentially concerns the lack of correlation between the moment indicator vector and the (implicit) score vector associated with the true model. Standard inferential tools such as LR and Wald test statistics no longer have the standard limiting normal or chi-square distributions. A number of methods have been proposed to ameliorate this problem, primarily related to score or Lagrange multiplier statistics as discussed in section 4; see, e.g., Kleibergen (2005), Guggenberger et al. (2012) and Otsu (2006). Newey and Windmeijer (2009) obtains the limiting properties of GMM and $(\mathrm{G}) \mathrm{EL}$ when there are many weak moments and, in particular, shows that the respective variance matrices are inflated in comparison to the standard variance matrix expression given in section 3 for efficient 2SGMM and GEL. 


\section{LITERATURE CITED}

Ai C, Chen X. 2003. Efficient estimation of models with conditional moment restrictions containing unknown functions. Econometrica 71:1795-1843.

Ali SM, Silvey SD. 1966. A general class of coefficient of divergence of one distribution from another. J. R. Stat. Soc. B 28:131-42.

Amemiya, T. 1974. The non-linear two stage least squares estimator. J. Econom. 2:105-1 10 .

Anatolyev S. 2005. GMM, GEL, serial correlation, and asymptotic bias. Econometrica 73:983-1002.

Anatolyev S, Gospodinov N. 2011. Methods for Estimation and Inference in Modern Econometrics. Boca Raton, FL: Chapman Hall/CRC.

Andrews DWK, and Shi X. (2013). Inference based on conditional moment inequalities. Econometrica 81:609-66.

Angrist JD, Krueger AB. 1991. Does compulsory school attendance affect schooling and earnings. Quart. J. Econ.106:979-1014.

Antoine B, Bonnal H, Renault, E. 2007. On the efficient use of the informational content of estimating equations: implied probabilities and Euclidean empirical likelihood. J. Econom. 138:461-87.

Baggerly K. 1998. Empirical likelihood as a goodness-of-fit measure. Biometrika 85:53547.

Bartlett MS. 1937. Properties of sufficiency and statistical tests. Proc. R. Soc. A 160:268-82. 
Beran R. 1977. Minimum Hellinger distance estimates for parametric models. Ann. Stat. 5:445-63.

Brown BW, Newey WK. 1998. Efficient semiparametric estimation of expectations. Econometrica 66:453-64.

Brown BW, Newey WK. 2002. Generalized method of moments, efficient bootstrapping, and improved inference. J. Bus. Econ. Stat. 20:507-17.

Canay IA. 2010. EL inference for partially identified models: large deviations optimality and bootstrap validity. J. Econom. 156: 408-25.

Chamberlain G. 1987. Asymptotic efficiency in estimation with conditional moment restrictions. J. Econom. 34:305-34.

Chaussé P. 2010. Computing generalized method of moments and generalized empirical likelihood with R. J. Stat. Software 34(11).

Chen J, Variyath AM, Abraham B. 2008. Adjusted empirical likelihood and its properties. J. Comput. Graph. Stat. 17:426-43.

Chen SX, Cui H-J. 2006. On Bartlett correction of empirical likelihood in the presence of nuisance parameters. Biometrika 93:215-20.

Chen SX, Cui H-J. 2007. On the second order properties of empirical likelihood with moment restrictions. J. Econom. 141:492-516.

Chen X, Pouzo D. 2009. Efficient estimation of semiparametric conditional moment models with possibly nonsmooth residuals. J. Econom. 152:46-60.

Chen X, Pouzo D. 2012. Estimation of nonparametric conditional moment models with possibly nonsmooth generalized residuals. Econometrica 80:277-321.

Chesher A, Smith RJ. 1997. Likelihood ratio specification tests. Econometrica 65:62746. 
Corcoran S. 1998. Bartlett adjustment of empirical discrepancy statistics. Biometrika 85:965-72.

Cragg JG. 1983. More efficient estimation in the presence of heteroscedasticity of unknown form. Econometrica 51:751-63.

Cressie N, Read T. 1984. Multinomial goodness-of-fit tests. J. R. Stat. Soc. B 46:44064.

Cribari-Neto F, Cordeiro GM. 1996. On Bartlett and Bartlett-type corrections. Econom. Rev. 15: 339-67.

Csiszar I. 1963. Eine informations theoretische ungleichungen und ihre anwendung auf den beweis der ergodicitat von Markoffschen ketten. Pub. Math. Inst. Hungarian Ac. Sci. 8:85-108.

Davidson R, MacKinnon JG. 2004. Econometric Theory and Methods. New York, NY: Oxford University Press.

DiCiccio T, Hall P, Romano J. 1991. Empirical likelihood is Bartlett-correctable. Ann. Stat. 19:1053-61.

Donald SG, Imbens GW, Newey WK. 2003. Empirical likelihood estimation and consistent tests with conditional moment restrictions. J. Econom. 117:55-93.

Fan Y, Gentry M, Li T. 2011. A new class of asymptotically efficient estimators for moment condition models. J. Econom. 162:268-77.

Goldberger AS. 1991. A Course in Econometrics. Cambridge, MA: Harvard University Press.

Ghosh JK. 1994. Higher Order Asymptotics. Vol. 4. NSF-CBMS Regional Conference Series in Probability and Statistics. Hayward, CA: Inst. Math. Stat. 
Greene WH. 2008. Econometric Analysis. Upper Saddle River, NJ: Pearson Prentice Hall. 6th ed.

Guggenberger P. 2008. Finite sample evidence suggesting a heavy tail problem of the generalized empirical likelihood estimator. Econom. Rev. 27:526-41.

Guggenberger P, Ramalho JJS, Smith RJ. 2012. GEL statistics under weak identification. J. Econom. 170:331-49.

Hall P, Horowitz JL. (1996). Bootstrap critical values for tests based on generalizedmethod-of-moment estimators. Econometrica 64:891-916.

Hansen LP. 1982. Large sample properties of generalized method of moments estimators. Econometrica 50:1029-54.

Hansen LP, Heaton J, Yaron A. 1996. Finite-sample properties of some alternative GMM estimators. J. Bus. Econ. Stat. 14:262-80.

Hjört NL, McKeague IW, Van Keilegom I. 2009. Extending the scope of empirical likelihood. Ann. Stat. 37:1079-111.

Hoeffding W. 1965. Asymptotically optimal tests for multinomial distributions. Ann. Math. Stat. 36:369-408.

Ichimura H. 1993. Semiparametric least squares (SLS) and weighted SLS estimation of single index models. J. Econom. 58:71-120.

Imbens GW. 1997. One-step estimators for over-identified generalized method of moments models. Rev. Econ. Stud. 64:359-83.

Imbens GW. 2002. Generalized method of moments and empirical likelihood. J. Bus. Econ. Stat. 20:493-506.

Imbens GW, Spady RH. 2002. Confidence intervals in generalized method of moments models. J. Econom. 107:87-98. 
Imbens GW, Spady RH. 2005. The performance of empirical likelihood and its generalizations. In Identification and Inference for Econometric Models, Essays in Honor of Thomas Rothenberg, ed. DWK Andrews and JH Stock, 10: 216-44. Cambridge: Cambridge University Press.

Imbens GW, Spady RH, Johnson P. 1998. Information theoretic approaches to inference in moment condition models. Econometrica 66:333-57.

Jing B-Y, Wood ATA. 1996. Exponential empirical likelihood is not Bartlett correctable. Ann. Stat. 24:365-9.

Kitamura Y. 1997. Empirical likelihood methods with weakly dependent processes. Ann. Stat. 25:2084-102.

Kitamura Y. 2001. Asymptotic optimality of empirical likelihood for testing moment restrictions. Econometrica 69:1661-72.

Kitamura Y. 2007. Empirical likelihood methods in econometrics: theory and practice. In Advances in Economics and Econometrics, Theory and Applications: Ninth World Congress of the Econometric Society, Vol. 3, Econom. Soc. Monograph Series, ESM 43, ed. RW Blundell, WK Newey and T Persson 7:174-237. Cambridge: Cambridge University Press.

Kitamura Y, Otsu T, Evdokimov K. 2013. Robustness, infinitesimal neighborhoods, and moment restrictions. Econometrica 81:1185-201.

Kitamura Y, Santos A, Shaikh AM. 2012. On the asymptotic optimality of empirical likelihood for testing moment restrictions. Econometrica 80:413-23.

Kitamura Y, Stutzer M. 1997. An information-theoretic alternative to generalized method of moments estimation. Econometrica 65:861-74.

Kitamura Y, Tripathi G, Ahn H. 2004. Empirical likelihood-based inference in conditional moment restriction models. Econometrica 72:1667-714. 
Kleibergen FR. 2005. Testing parameters in GMM without assuming that they are identified. Econometrica 73:1103-23.

Liu Y, Chen J. 2010. Adjusted empirical likelihood with high-order precision. Ann. Stat. 38:1341-62.

Manski CF. (1988). Analog Estimation Methods in Econometrics. NewYork, NY: Chapman and Hall.

Matsushita Y, Otsu T. 2013. Second-order refinement of empirical likelihood for testing overidentifying restrictions. Econom. Theory 29:324-53.

Mittelhammer RC, Judge GG, Schoenberg R. 2005. Empirical evidence concerning the finite sample performance of EL-type structural equation estimation and inference methods. In Identification and Inference for Econometric Models, Essays in Honor of Thomas Rothenberg, ed. DWK Andrews and JH Stock, 12: 282-305. Cambridge: Cambridge University Press.

Newey WK, Ramalho JJS, Smith RJ. 2005. Asymptotic bias for GMM and GEL estimators with estimated nuisance parameters. In Identification and Inference for Econometric Models, Essays in Honor of Thomas Rothenberg, ed. DWK Andrews and JH Stock, 11: 245-81. Cambridge: Cambridge University Press.

Newey WK, Smith RJ. 2004. Higher order properties of GMM and generalized empirical likelihood estimators. Econometrica 72:219-55.

Newey WK, Windmeijer F. 2009. Generalized method of moments with many weak moment conditions. Econometrica 77:687-719.

Otsu T. 2006. Generalized empirical likelihood inference for nonlinear and time series models under weak identification. Econom. Theory 22:513-27.

Otsu T. 2007. Penalized empirical likelihood estimation of semiparametric models. J. Mult. Anal. 98:1923-54. 
Otsu T. 2011. Empirical likelihood estimation of conditional moment restriction models with unknown functions. Econom. Theory 27:8-46.

Owen A. 1988. Empirical likelihood ratio confidence intervals for a single functional. Biometrika 75:237-49.

Owen A. 1990. Empirical likelihood ratio confidence regions. Ann. Stat. 18:90-120.

Owen A. 2001. Empirical Likelihood. New York, NY: Chapman and Hall.

Powell J, Stock JH, Stoker T. .1989. Semiparametric estimation of index coefficients. Econometrica 57:1403-30.

Powell MJD. 1981. Approximation Theory and Methods. Cambridge: Cambridge University Press.

Qin J, Lawless J. 1994. Empirical likelihood and general estimating equations. Ann. Stat. 22:300-25.

Ramalho JJS. 2005. Small sample bias of alternative estimation methods for moment condition models: Monte Carlo evidence for covariance structures. Stud. Nonlin. Dyn. Econom. 9:1-20.

Reiersol O. 1941. Confluence analysis by means of lag moments and other methods of confluence analysi. Econometrica 9:1-23.

Reiersol O. 1945. Confluence analysis by means of instrumental sets of variables. Ark. Matematik, Astr. Och Fysik 32A:1-119.

Robinson PM. 1988a. The stochastic difference between econometric estimators. Econometrica 56:531-48.

Robinson PM. 1988b. Root-n-consistent semiparametric regression. Econometrica 56:93154. 
Sargan JD. 1958. The estimation of economic relationships using instrumental variables. Econometrica 26:393-415.

Sargan JD. 1959. The estimation of relationships with autocorrelated residuals by the use of the instrumental variables. J. R. Stat. Soc. B 21:91-105.

Schennach SM. 2007. Point estimation with exponentially tilted empirical likelihood. Ann. Stat. 35:634-72.

Shen X. 1997. On methods of sieves and penalization. Ann. Stat. 25: 2555-91.

Smith RJ. 1997. Alternative semi-parametric likelihood approaches to generalized method of moments estimation. Econ. J. 107: 503-19.

Smith RJ. 2000. Empirical likelihood estimation and inference. In Applications of Differential Geometry to Econometrics, ed. P Marriott and M Salmon, 4:119-50. Cambridge: Cambridge University Press.

Smith RJ. 2005. Automatic positive semi-definite HAC covariance matrix and GMM estimation. Econom. Theory 21:158-70.

Smith RJ. 2007a. Efficient information theoretic inference for conditional moment restrictions. J. Econom. 138:430-60.

Smith RJ. 2007b. Local GEL estimation with conditional moment restrictions. In The Refinement of Econometric Estimation and Test Procedures: Finite Sample and Asymptotic Analysis, ed. GDA Phillips and E Tzavalis, 4:100-22. Cambridge: Cambridge University Press.

Smith RJ. 2007c. Weak instruments and empirical likelihood: a discussion of the papers by D.W.K. Andrews and J.H. Stock and Y. Kitamura. In Advances in Economics and Econometrics, Theory and Applications: Ninth World Congress of the Econometric Society, Vol. 3, Econom. Soc. Monograph Series, ESM 43, ed. RW Blundell, WK Newey and T Persson, 8:238-60. Cambridge: Cambridge University Press. 
Smith RJ. 2011. GEL criteria for moment condition models. Econom. Theory 27:1192235.

Tripathi G, Kitamura Y. 2003. Testing conditional moment restrictions. Ann. Stat. 31:2059-95.

Wright PG. 1928. The Tariff on Animal and Vegetable Oils. New York: Macmillan.

Wright S. 1925. Corn and hog correlations. US Dept. of Agric. Bull. 1300:1-60.

Zhang J, Gijbels I. 2003. Sieve empirical likelihood and extensions of generalized least squares. Scand. J. Stat. 30:1-24. 